\title{
A Dynamic Model of a Cantilever Beam with a Closed, Embedded Horizontal Crack Including Local Flexibilities at Crack Tips
}

J. Liu ${ }^{\mathrm{a}, \mathrm{b}, \mathrm{c}^{*}}$, W.D. Zhu ${ }^{\mathrm{c}^{*}}$, P.G. Charalambides ${ }^{\mathrm{c}}$, Y.M. Shao ${ }^{\mathrm{a}}$, Y.F. Xu ${ }^{\mathrm{c}}$, and X.M. Fang ${ }^{\mathrm{c}}$

a) State Key Laboratory of Mechanical Transmission, Chongqing University, Chongqing, 400030, P.R. China

b) College of Mechanical Engineering, Chongqing University, Chongqing, 400030,

$$
\text { P.R. China }
$$

c) Department of Mechanical Engineering, University of Maryland Baltimore County, Baltimore, MD 21250, U.S.A.

* Corresponding author. Tel.: 410-455-3394 (W.D. Zhu), +86-023-65112520 (J. Liu). E-mail addresses: wzhu@umbc.edu (W.D. Zhu), jliu@ cqu.edu.cn (J. Liu).

\begin{abstract}
As one of major failure modes of mechanical structures subjected to periodic loads, embedded cracks due to fatigue can cause catastrophic failure of machineries. Understanding the dynamic characteristics of a structure with an embedded crack is helpful for early crack detection and diagnosis. In this work, a new three-segment beam model with local flexibilities at crack tips is developed to investigate the vibration of a cantilever beam with a closed, fully embedded horizontal crack, which is assumed to be not located at its clamped or free end or distributed near its top or bottom side. The three-segment beam model is assumed to be a linear elastic system, and it does not account for the nonlinear crack closure effect; the top and bottom
\end{abstract}


segments always stay in contact at their interface during the beam vibration. It can model effects of local deformations in the vicinity of the crack tips, which cannot be captured by previous methods in the literature. The middle segment of the beam containing the crack is modeled by a mechanically consistent, reduced bending moment. Each beam segment is assumed to be an Euler-Bernoulli beam, and the compliances at the crack tips are analytically determined using a $J$-integral approach and verified using commercial finite element software. Using compatibility conditions at the crack tips and the transfer matrix method, the nature frequencies and mode shapes of the cracked cantilever beam are obtained. The three-segment beam model is used to investigate the effects of local flexibilities at crack tips on the first three natural frequencies and mode shapes of the cracked cantilever beam. A stationary wavelet transform (SWT) method is used to process the mode shapes of the cracked cantilever beam; jumps in single-level SWT decomposition detail coefficients can be used to identify the length and location of an embedded horizontal crack.

Keywords: Vibration; Cracked cantilever beam; Fully embedded horizontal crack; Closed crack; J-integral; Three-segment beam model

\section{Introduction}

Embedded cracks are one of major failure modes of mechanical structures subjected to periodic loads. The dynamic characteristics and the safety of machineries are greatly affected by cracks due to fatigue. To prevent catastrophic failure of 
machineries, mechanical structure monitoring for early crack detection and diagnosis is an important task for industrial maintenance.

When a crack occurs in a structure, its static and dynamic characteristics such as the stiffness, natural frequencies, mode shapes, damping, and vibration amplitudes will be changed [1,2]. An investigation of changes in the static and dynamic characteristics makes it possible to detect a crack in a structure [1-4]. While mode shapes and damping are more sensitive to the existence of a crack in a structure than natural frequencies in practice, magnitudes of changes in the natural frequencies are also functions of the severity and location of the cack in the structure [2,5-10]. Many research works determine damage severity of beam structures using analytical, numerical, and experimental methods. For an open crack, damage detection depends on changes in the static and dynamic characteristics. For a breathing crack, it depends on nonlinear dynamic characteristics, such as periodical structural stiffness variation, modulation frequencies, and higher harmonics, which was discussed in Ref. [11].

As one of accurate and comprehensive methods, a modeling and simulation method can predict the dynamic characteristics of a cracked structure and provide some guidance to early detection of crack failure. Many previous works focused on an edge crack $[9,12-24]$ and multiple edge cracks $[3,10,25-30]$ in cantilever or simply supported beams. Some researchers have studied delaminations in beam structures [16,31-46]. While some studies $[33,34,41,43,46]$ are focused on detection of delamination in a laminated material, some $[4,16,31,32,35-38,40,42,44,45]$ are focused on modeling delaminations in beam structures based on analytical methods, 
the finite element (FE) method, and experimental methods. The compatibility conditions at the junctions are formulated as changes in the axial forces and bending moments there $[16,31,32,35-37,39,40,42,44]$, which cannot describe local flexibilities at crack tips due to the presence of a crack. Wang and Qiao [38] used a shear compliance coefficient at a crack tip to describe the crack tip deformation for a simply-supported end-notched beam specimen. Qiao and Chen [45] used the model in Ref. [38] to study deformations at delamination tips in a clamped and a simply supported bi-layer composite beam with an interface delamination. Cantilever beam structures with embedded cracks have not been discussed in the literature.

In this work, a new three-segment beam model with local flexibilities at crack tips is developed to investigate the vibration of a cantilever beam with a closed, fully embedded horizontal crack, which is assumed to be not located near its clamped or free end or distributed near its top or bottom side. For the closed, fully embedded horizontal crack, the top and bottom segments always stay in contact at their interface and have the same transverse displacements; they can slide over each other in the axial direction except at their ends. Such a crack can occur in a layered structure prone to delamination. While the beam is assumed to be homogeneous here, the methodology developed in this work can be extended to laminates with homogeneous layers, whose material properties are not functions of spatial variables. Hence, the three-segment beam model is assumed to be a linear elastic system and does not account for the nonlinear crack closure effect. The proposed model can describe the effects of local deformations in the vicinity of the crack tips, which cannot be 
captured by previous analytical methods in the literature. The middle segment of the beam containing the crack has a mechanically consistent, reduced bending moment. This work builds on parallel studies in Ref. [47] where the macro-mechanics of a cantilever beam with an embedded horizontal crack under a static load is addressed. Each beam segment is assumed to be an Euler-Bernoulli beam in this work, which implies that it is slender. However, the methodology developed here can be extended to the case where each beam segment is modeled as a Timoshenko beam. Compliances at the crack tips are analytically determined using a $J$-integral approach [48]. The $J$-integral approach may not be used to analyze a breathing, embedded crack with opening and closing states, or a crack located near the clamped or free end of the beam, or distributed near its top or bottom side. Using compatibility conditions at the crack tips and the transfer matrix method [18,27,49], the natural frequencies and mode shapes of the cracked cantilever beam are obtained. Since the FE method has been widely used in deformation and vibration studies of beams with cracks $[1,22,42,50,51-56]$, the $J$-integral and stress state results from the analytical method are verified using commercial FE software [57]. A more detailed comparison of the $J$-integral results using the analytical and FE methods is presented in Ref. [47].

The new three-segment beam model is used to investigate the effects of local flexibilities at crack tips on the first three natural frequencies and mode shapes of the cracked cantilever beam. The results show that the model put forward here is an improvement over the related one, where crack-induced rotational flexibilities of cross-sections of the beam at the crack tips are not considered. As will be 
demonstrated later in this study, inclusion of local flexibilities at the crack tips can model kinks in mode shapes there. A stationary wavelet transform (SWT) method $[58,59]$ is used to process mode shapes of the cracked cantilever beam; it is shown that jumps in single-level SWT decomposition detail coefficients can be used to identify the location and size of an embedded horizontal crack. This study is a first step towards modeling and detecting a slant crack in a beam structure.

\section{Crack-induced local flexibilities at crack tips}

A uniform Euler-Bernoulli cantilever beam of length $L$, height $h$, and width $b$ with a closed, fully embedded horizontal crack is shown in Figure 1, where $P$ is an applied force, $X_{0}$ and $X_{3}$ are the fixed and free ends of the beam, respectively, and $X_{1}$ and $X_{2}$ represent the end points of the crack relative to the global $X-Y$ coordinates. The crack length is $L_{2}$ and the crack depth from the top surface of the beam is $h_{1}$ with $0<$ $h_{1}<h$. It is assumed that the center of the crack is located at $X_{c}$ with $L_{2} / 2<X_{c}<L-L_{2} / 2$. The beam is divided into three segments of lengths $L_{1}, L_{2}$, and $L_{3}$, which are separated by the end points of the crack.
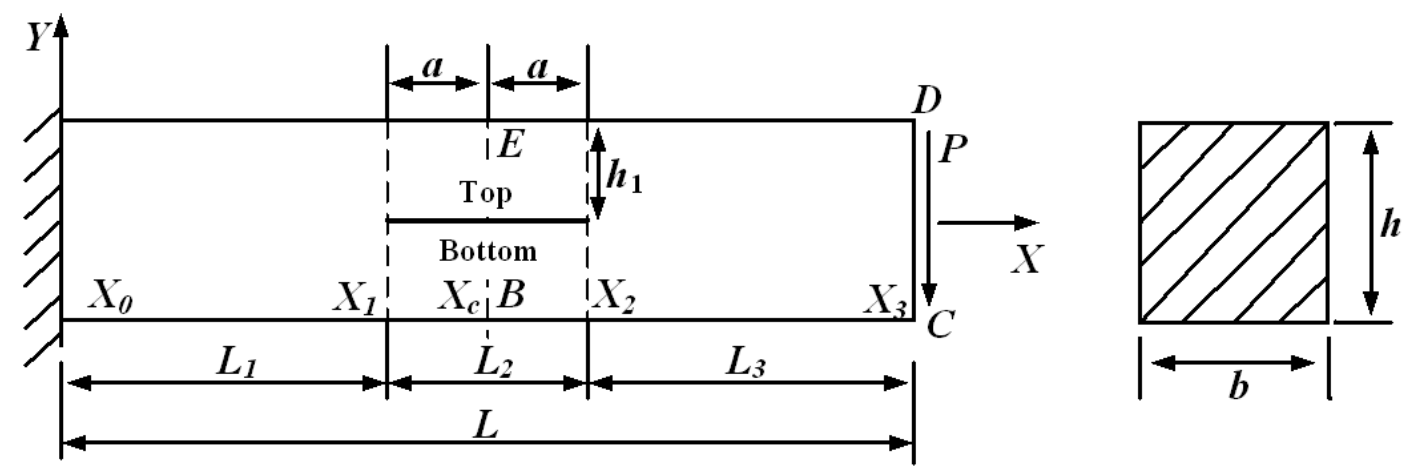

Figure 1. Schematic of a cantilever beam with a closed, fully embedded horizontal crack. 
The equivalent crack-induced rotational flexibilities of cross-sections of the beam at the two crack tips are analytically determined here using a $J$-integral approach. The beam is divided into two parts at the center of the crack, as shown in Figure 2, where $P_{1}$ and $P_{2}$ are the shear forces on the edges EF and $\mathrm{AB}$, respectively, $M_{1}$ and $M_{2}$ are the bending moments at $\mathrm{EF}$ and $\mathrm{AB}$, respectively, and $N_{1}$ and $N_{2}$ are the axial forces acting at the centroids of the top and bottom cross-sections of the beam at the center of the crack, respectively. The $J$-integrals along the contours of the left (FEDCBA) and right $(\mathrm{ABCDEF})$ parts of the beam are determined in what follows. The local coordinates for the left and right parts of the beam are shown in Figure 2.

(a)

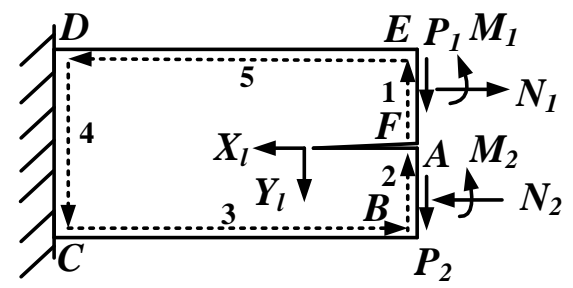

(b)

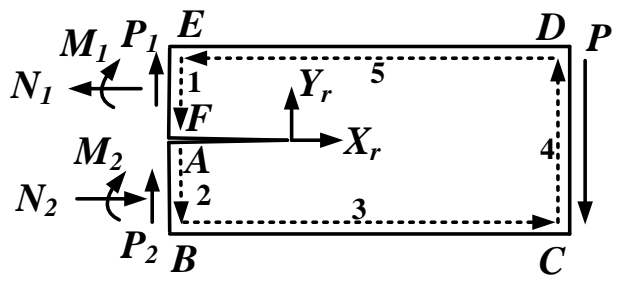

Figure 2. Two parts of the cracked beam and paths used in determining the $J$-integrals along the contours of the (a) left (FEDCBA) and (b) right (ABCDEF) parts of the beam.

According to Rice's method [48], a J-integral is given by

$$
J=\int_{\Gamma}\left(W d Y-T_{i} \frac{\partial u_{i}}{\partial x} d s\right)
$$

where the Einstein summation convention is used; $W$ is the strain energy density; $\Gamma$ is a curve surrounding a crack tip; $T_{i}=\sigma_{i j} n_{j}$ are components of the traction vector, in which $\sigma_{i j}$, with $i, j=1,2$ for a planar problem, are stress components, and $n_{j}$ are components of the outward normal along $\Gamma ; u_{i}$ are components of the displacement 
vector; $d Y$ is a length element along the $Y_{r}$ or $Y_{l}$ axis in the local coordinates, as shown in Figure 2; and $d s$ is an arc length element along $\Gamma$. For the right part of the beam in Figure 2(b), the $J$-integral along the contour ABCDEF is the sum of line integrals along segments $\mathrm{AB}, \mathrm{BC}, \mathrm{CD}, \mathrm{DE}$, and $\mathrm{EF}$ of the contour:

$$
J^{r}=J_{A B}^{r}+J_{B C}^{r}+J_{C D}^{r}+J_{D E}^{r}+J_{E F}^{r}
$$

where the superscript $r$ denotes the right part. For segments $\mathrm{BC}$ and $\mathrm{DE}, d Y=0$ and $T_{i}=0$; hence

$$
J_{B C}^{r}=0, \quad J_{D E}^{r}=0
$$

For segment $\mathrm{AB}$, one has

$$
J_{A B}^{r}=\int_{A B}\left(W_{A B}^{r} d Y-T_{i} \frac{\partial u_{i}}{\partial x} d s\right)=\int_{A B}\left(-W_{A B}^{r} d s-\left(T_{1} \frac{\partial u_{1}^{r}}{\partial X}+T_{2} \frac{\partial u_{2}^{r}}{\partial X}\right) d s\right)
$$

where

$$
W_{A B}^{r}=\frac{1}{2} \sigma_{x x 2}^{r} \varepsilon_{x x 2}^{r}+\frac{1}{2} \tau_{x y 2}^{r} \gamma_{x y 2}^{r}
$$

in which $\sigma_{x x 2}^{r}$ and $\tau_{x y 2}^{r}$ are the normal and shear stresses on segment $\mathrm{AB}$, respectively, $\varepsilon_{x x 2}^{r}$ and $\gamma_{x y 2}^{r}$ are the normal and shear strains of segment $\mathrm{AB}$, respectively, and the subscript 2 denotes the stress and strain components associated with segment $\mathrm{AB}$. They are given by

$$
\begin{aligned}
& \sigma_{x x 2}^{r}=\frac{N_{2}}{A_{b}}-\frac{M_{2}}{I_{b}} Y, \varepsilon_{x x 2}^{r}=\frac{\partial u_{1}^{r}}{\partial X}=\frac{N_{2}}{E A_{b}}-\frac{M_{2}}{E I_{b}} Y \\
& \tau_{x y 2}^{r}=\frac{P_{2} S(Y)}{I_{b} t_{1}}, \gamma_{x y 2}^{r}=\frac{\tau_{x y 2}^{r}}{G}=\frac{\partial u_{1}^{r}}{\partial Y}+\frac{\partial u_{2}^{r}}{\partial X}=\frac{P_{2} S(Y)}{G I_{b} t_{1}}
\end{aligned}
$$

where $E$ and $G$ are the elastic and shear moduli of the beam, respectively, $A_{\mathrm{b}}$ is the cross-sectional area of the bottom segment of $X_{1} X_{2}, I_{\mathrm{b}}$ is the cross-sectional area moment of inertia of the bottom segment of $X_{1} X_{2}$ about its centroidal axis, $X$ and $Y$ are 
the displacements in the $X$ and $Y$ directions, respectively, $S(Y)=Y d A$, in which $d A$ is an element area, and $u_{1}^{r}$ and $u_{2}^{r}$ are the deflections of the bottom segment of $X_{1} X_{2}$ in the $X$ and $Y$ directions, respectively. The relationship between the deflections in the $X$ and $Y$ directions is [60]

$$
u_{1}^{r}=u_{1}^{0 r}-\frac{\partial u_{2}^{0 r}}{\partial X} Y
$$

where the superscript 0 denotes deflections along the centroidal axis of the bottom segment of $X_{1} X_{2}$. Differentiating equation (7) with respect to $Y$ yields

$$
\frac{\partial u_{1}^{r}}{\partial Y}=-\frac{\partial^{2} u_{2}^{0 r}}{\partial X \partial Y} Y-\frac{\partial u_{2}^{0 r}}{\partial X}=-\frac{\partial u_{2}^{0 r}}{\partial X}=-\frac{\partial u_{2}^{r}}{\partial X}
$$

where $\frac{\partial^{2} u_{2}^{0 r}}{\partial X \partial Y}=0$ since the slope of the deflection along the centroidal axis of the bottom segment of $X_{1} X_{2}$ does not depend on $Y$. As discussed in Ref. [47], for the cracked cantilever beam, the deflection angle of the cross-section $\mathrm{AB}$ can be assumed to be the sum of the deflection angle of the cross-section at $X_{1}$ relative to that at $X_{0}$ and the deflection angle of the cross-section at $X_{\mathrm{c}}$ relative to that at $X_{1}$ :

$$
\frac{\partial u_{2}^{r}}{\partial X}=-\frac{P L_{1}^{2}}{2 E I}-\frac{P\left(L-L_{1}\right) L_{1}}{E I}-\frac{P_{2} a^{2}}{2 E I_{b}}+\frac{M_{2} a}{E I_{b}}
$$

where $a=0.5 L_{2}$ (Figure 1). Then

$$
\gamma_{x y 2}^{r}=\frac{\partial u_{2}^{r}}{\partial X}=-\frac{P L_{1}^{2}}{2 E I}-\frac{P\left(L-L_{1}\right) L_{1}}{E I}-\frac{P_{2} a^{2}}{2 E I_{b}}+\frac{M_{2} a}{E I_{b}}
$$

The traction vector components for segment $\mathrm{AB}$ are given by

$$
T_{1}=\sigma_{11} n_{1}+\sigma_{12} n_{2}=-\sigma_{11}, T_{2}=\sigma_{21} n_{1}+\sigma_{22} n_{2}=-\sigma_{21}
$$

Use of equations (5)-(10) in equation (4) yields 


$$
\begin{aligned}
J_{A B}^{r} & =\int_{B A} \frac{1}{2} \sigma_{x x 2}^{r} \varepsilon_{x x 2}^{r} d Y+\int_{B A} \tau_{x y 2} \frac{\partial u_{2}^{r}}{\partial X} d Y \\
& =\frac{N_{2}^{2}}{2 E A_{b}}+\frac{M_{2}^{2}}{2 E I_{b}}+\frac{P P_{2} L_{1}^{2}}{2 E I}+\frac{P P_{2}\left(L-L_{1}\right) L_{1}}{E I}+\frac{P_{2}^{2} a^{2}}{2 E I_{b}}+\frac{P_{2} M_{2} a}{E I_{b}}
\end{aligned}
$$

Similarly, the line integral along segment EF is

$$
J_{E F}^{r}=\frac{N_{1}^{2}}{2 E A_{t}}+\frac{M_{1}^{2}}{2 E I_{t}}+\frac{P P_{1} L_{1}^{2}}{2 E I}+\frac{P P_{1}\left(L-L_{1}\right) L_{1}}{E I}+\frac{P_{1}^{2} a^{2}}{2 E I_{t}}+\frac{P_{1} M_{1} a}{E I_{t}}
$$

where $A_{t}$ is the cross-section area of the top segment of $X_{1} X_{2}$, and $I_{t}$ is the cross-sectional area moment of inertia of the top segment of $X_{1} X_{2}$ about its centroidal axis. For segment CD,

$$
J_{C D}^{r}=\int_{C D} W_{C D}^{r} d Y-T_{i} \frac{\partial u_{i}}{\partial X} d s=\int_{C D}\left(W_{C D}^{r}-\left(T_{1} \frac{\partial u_{1}^{r}}{\partial X}+T_{2} \frac{\partial u_{2}^{r}}{\partial X}\right)\right) d s
$$

where

$$
W_{C D}^{r}=\frac{1}{2} \sigma_{x x 4}^{r} \varepsilon_{x x 4}^{r}+\frac{1}{2}\left(\tau_{x y 4}^{r} \varepsilon_{x y 4}^{r}+\tau_{y x 4}^{r} \varepsilon_{y x 4}^{r}\right)=\frac{1}{2} \sigma_{x x 4}^{r} \varepsilon_{x x 4}^{r}+\frac{1}{2} \tau_{x y 4}^{r} \gamma_{x y 4}^{r}
$$

in which the subscript 4 denotes the stress and strain components associated with segment $\mathrm{CD}$. The normal stress, normal strain, shear stress, and shear strain for segment $\mathrm{CD}$ are given by

$$
\begin{aligned}
& \sigma_{x x 4}^{r}(y)=0, \quad \varepsilon_{x x 4}^{r}(y)=\frac{\sigma_{x x 4}^{r}}{E}=\frac{\partial u_{1}^{r}}{\partial X}=0 \\
& \tau_{x y 4}^{r}=\frac{P S(Y)}{I t_{1}}, \quad \gamma_{x y 4}^{r}=\frac{\tau_{x y 4}^{r}}{G}=\frac{\partial u_{1}^{r}}{\partial Y}+\frac{\partial u_{2}^{r}}{\partial X}=\frac{P S(Y)}{G I t_{1}}
\end{aligned}
$$

The rotational angle of the cross-section CD is assumed to be the sum of the rotational angle of the cross-section at $X_{1}$ relative to that at $X_{0}$, the rotational angle of the cross-section at $X_{2}$ relative to that at $X_{1}$ for either the top or bottom segment of $X_{1} X_{2}$ since the top and bottom segments have the same rotational angles at $X_{1}$ and $X_{2}$, and the rotational angle of the cross-section at $X_{3}$ relative to that at $X_{2}$ : 


$$
\begin{aligned}
\frac{\partial u_{2}^{r}}{\partial X} & =-\frac{P L_{1}^{2}}{2 E I}-\frac{P\left(L-L_{1}\right) L_{1}}{E I}+\frac{2 a\left(M_{1}+P_{1} b\right)}{E I_{t}}-\frac{P_{1}(2 a)^{2}}{2 E I_{t}}-\frac{P L_{3}^{2}}{2 E I} \\
& =-\frac{P L_{1}^{2}}{2 E I}-\frac{P\left(L-L_{1}\right) L_{1}}{E I}+\frac{2 M_{1} a}{E I_{t}}-\frac{P L_{3}^{2}}{2 E I}
\end{aligned}
$$

The traction vector components $T_{i}$ for segment $\mathrm{CD}$ are given by

$$
T_{1}=\sigma_{11} n_{1}+\sigma_{12} n_{2}=\sigma_{11}, T_{2}=\sigma_{21} n_{1}+\sigma_{22} n_{2}=\sigma_{21}
$$

Use of equations (15)-(18) in equation (14) yields

$$
J_{C D}^{r}=\int_{C D}\left(W_{C D}^{r}-\left(T_{1} \frac{\partial u_{1}^{r}}{\partial X}+T_{2} \frac{\partial u_{2}^{r}}{\partial X}\right)\right) d s=-\int_{C D} \tau_{x y 4}^{r} \frac{\partial u_{2}^{r}}{\partial X} d s=\frac{\partial u_{2}^{r}}{\partial X} P
$$

By a static FE analysis [31,47] and experimental validation [31], one can find that the curvature of the static deflection of a cantilever beam at the center of a horizontal crack is the same as that of the corresponding beam without the crack:

$$
\frac{\partial^{2} Y_{t}}{\partial X^{2}}=\frac{\partial^{2} Y_{b}}{\partial X^{2}}=\frac{\partial^{2} Y_{h}}{\partial X^{2}}
$$

where $Y_{t}$ and $Y_{b}$ are the displacements of the top and bottom segments of $X_{1} X_{2}$, respectively, and $Y_{h}$ is the displacement of the corresponding beam without the crack. Hence

$$
\frac{M_{1}}{E I_{t}}=\frac{M_{2}}{E I_{b}}=\frac{M_{c}}{E I}
$$

Consequently, one has

$$
M_{1}=\frac{I_{t} M_{c}}{I}, \quad M_{2}=\frac{I_{b} M_{c}}{I}
$$

By moment balance of the right part of the beam at point $\mathrm{B}$, one has

$$
M_{c}-M_{1}-M_{2}+\frac{N_{2}\left(h-h_{1}\right)}{2}+N_{1}\left(h-\frac{h_{1}}{2}\right)=0
$$

where $M_{c}=-P\left(L-X_{c}\right)$ and $N_{2}=-N_{1}$. Hence 


$$
N_{2}=-N_{1}=-\frac{2 M_{c}}{h}\left(1-\frac{I_{t}+I_{b}}{I}\right)=\frac{6 h_{1} P\left(L-X_{c}\right)\left(h-h_{1}\right)}{h^{3}}
$$

By force balance of the right part of the beam in the $Y$ direction, one has

$$
P_{1}+P_{2}=P
$$

By deflection compatibility of the beam at the cross-section at $X_{2}$, i.e., the deflection along the centroidal axis of the top segment of $X_{1} X_{2}$ in the $Y$ direction at $X_{2}$ relative to that at $X_{1}$ is the same as the deflection along the centroidal axis of the bottom segment of $X_{1} X_{2}$ in the $Y$ direction at $X_{2}$ relative to that at $X_{1}$, one has

$$
\frac{\left(M_{1}+P_{1} a\right)(2 a)^{2}}{2 E I_{t}}-\frac{P_{1}(2 a)^{3}}{3 E I_{t}}=\frac{\left(M_{2}+P_{2} a\right)(2 a)^{2}}{2 E I_{b}}-\frac{P_{2}(2 a)^{3}}{3 E I_{b}}
$$

By equations (24)-(26), one has

$$
P_{1}=\frac{I_{t}}{I_{t}+I_{b}} P, \quad P_{2}=\frac{I_{b}}{I_{t}+I_{b}} P
$$

Substituting equations (3), (12), (13), (17), (19), (21), (23), and (27) into equation (2) yields

$$
\begin{aligned}
J^{r}= & J_{A B}^{r}+J_{B C}^{r}+J_{C D}^{r}+J_{D E}^{r}+J_{E F}^{r} \\
= & \frac{6\left(L-X_{c}\right)^{2} P^{2}}{E h^{3}}+\frac{6\left(X_{c}-a\right)\left(2 L-X_{c}+a\right)}{E h^{3}} P^{2}-\frac{6 L^{2}}{E h^{3}} P^{2} \\
& +\frac{6 a^{2}}{E\left[h_{1}^{3}+\left(h-h_{1}\right)^{3}\right]} P^{2}+\frac{12 a\left(L-X_{c}\right)}{E h^{3}} P^{2} \\
= & \left(\frac{h^{3}}{h_{1}^{3}+\left(h-h_{1}\right)^{3}}-1\right) \frac{6 a^{2} P^{2}}{E h^{3}}
\end{aligned}
$$

The change in the strain energy caused by the horizontal crack is [19]

$$
U_{c}^{r}=\int_{0}^{a} J^{r}(a) d a
$$

By Castigliano's theorem $[22,23,61,62]$, the additional rotation $\theta$ caused by the horizontal crack at the cross-section at $X_{2}$ can be obtained: 


$$
\theta_{r}=\frac{\partial}{\partial P}\left[\int_{0}^{a} J^{r}(a) d a\right] \frac{\frac{1}{\partial M_{r}}}{\partial P}
$$

where $M_{r}=-P\left(L-\left(x_{C}+a\right)\right)$ is the bending moment at the cross-section at $X_{2}$. By equations (28) and (30), the equivalent rotational flexibility coefficient of the cross-section at $X_{2}$ is

$$
c_{2}=\frac{\partial \theta_{r}}{\partial P} \frac{1}{\frac{\partial M_{r}}{\partial P}}=\frac{b a^{3}}{3 E I\left(L-\left(X_{c}+a\right)\right)^{2}}\left(\frac{h^{3}}{h_{1}^{3}+\left(h-h_{1}\right)^{3}}-1\right)
$$

The rotational flexibility of the cross-section at $X_{2}$ can be modeled by a compliance $c_{2}$.

Proceeding in a similar manner, one can obtain the $J$-integral along the contour FEDCBA of the left part of the beam (see the Appendix for more details):

$$
J^{l}=J_{A B}^{l}+J_{B C}^{l}+J_{C D}^{l}+J_{D E}^{l}+J_{E F}^{l}=\left(\frac{h^{3}}{h_{1}^{3}+\left(h-h_{1}\right)^{3}}-1\right) \frac{6 a^{2} P^{2}}{E h^{3}}
$$

which is the same as that in equation (28). The additional rotation $\theta$ caused by the horizontal crack at the cross-section at $X_{1}$ is

$$
\theta_{l}=\frac{\partial}{\partial P}\left[\int_{0}^{b} J^{l}(a) d a\right] \frac{1}{\frac{\partial M_{l}}{\partial P}}
$$

where $M_{l}=-P\left(L-\left(x_{C}-a\right)\right)$ is the bending moment at the cross-section at $X_{1}$. The equivalent rotational flexibility of the cross-section at $X_{1}$ is

$$
c_{1}=\frac{\partial \theta_{l}}{\partial P} \frac{1}{\frac{\partial M_{l}}{\partial P}}=\frac{b a^{3}}{3 E I\left(L-\left(X_{c}-a\right)\right)^{2}}\left(\frac{h^{3}}{h_{1}^{3}+\left(h-h_{1}\right)^{3}}-1\right)
$$

The rotational flexibility of the cross-section at $X_{1}$ can be modeled by a compliance $c_{1}$. It can be seen from equations (31) and (34) that $c_{1}=c_{2}=0$ when $a=0$ or $h_{1}=0$, as 
expected, and $c_{1}>c_{2}$. The nondimensional compliances are $c_{1}^{*}=E I c_{1} / L t_{1}^{2}$ and $c_{2}^{*}=E I c_{2} / L t_{1}^{2}$.

\section{Free vibration analysis of a three-segment beam model}

When the crack remains closed, the top and bottom segments of $X_{1} X_{2}$ have the same transverse displacements, but they can slide over each other in the axial direction except at their ends [16]. Hence the top and bottom segments of $X_{1} X_{2}$ can be considered as one beam segment whose cross-sectional area moment of inertia is the sum of those of the top and bottom segments. Under the assumption that the ratio of the length of each beam segment to its height is larger than ten, the cantilever beam with a closed, embedded horizontal crack can be modeled as a three-segment beam with local flexibilities at $X_{1}$ and $X_{2}$, whose compliances are $c_{1}$ and $c_{2}$, respectively, as shown in Figure 3, and each beam segment can be modeled as an Euler-Bernoulli beam. The transverse displacement of the $k$ th $(k=1,2,3)$ segment of the beam is denoted by $Y_{k}(X, T)$ with $X_{k-1}<X<X_{k}$.

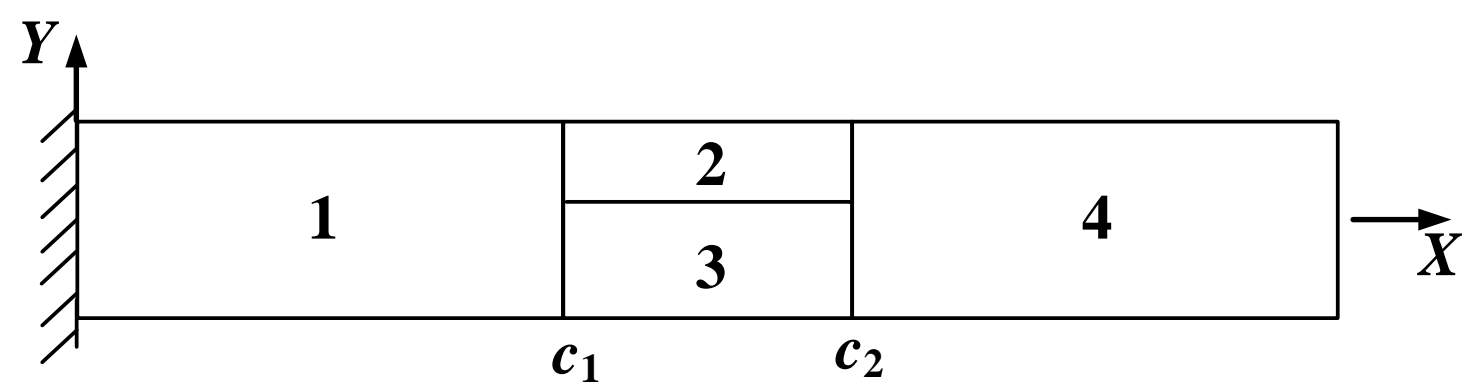

Figure 3. Schematic of a three-segment beam model with compliances $c_{1}$ and $c_{2}$ at crack tips.

According to Euler Bernoulli beam theory [12,16,25-28], the equations of motion of the three beam segments are given by 


$$
\begin{aligned}
& E I \frac{\partial^{4} Y_{k}(X, T)}{\partial X^{4}}+\rho A \frac{\partial^{2} Y_{k}(X, T)}{\partial T^{2}}=0, \quad X_{k-1}<X<X_{k}, \quad k=1,3 \\
& E\left(I_{t}+I_{b}\right) \frac{\partial^{4} Y_{2}(X, T)}{\partial X^{4}}+\rho A \frac{\partial^{2} Y_{2}(X, T)}{\partial T^{2}}=0, \quad X_{1}<X<X_{2}
\end{aligned}
$$

where $I$ is the cross-sectional area moment of inertia of the first and third segments of the beam, $\rho$ is their mass density, $A$ is their cross-sectional area, and $A_{t}+A_{b}=A$ has been used in equation (36). Note that $I_{t}+I_{b}<I$; hence the middle beam segment has a reduced cross-sectional area moment of inertia. Consequently, $E\left(I_{t}+I_{b}\right)<E I$; hence the middle beam segment has a reduced bending moment. The boundary conditions of the beam are $[26,27]$

$$
Y_{1}(0, T)=Y_{1}^{\prime}(0, T)=0, \quad Y_{3}^{\prime \prime}(0, T)=Y_{3}^{\prime \prime \prime}(L, T)=0
$$

The continuity conditions at the cross-sections at $X_{1}$ and $X_{2}$ are [16]

$$
\begin{aligned}
& Y_{1}\left(X_{1}^{-}, T\right)=Y_{2}\left(X_{1}^{+}, T\right) \\
& Y_{2}\left(X_{2}^{-}, T\right)=Y_{3}\left(X_{2}^{+}, T\right) \\
& Y_{1}^{\prime}\left(X_{1}^{-}, T\right)=Y_{2}^{\prime}\left(X_{1}^{+}, T\right) \\
& Y_{3}^{\prime}\left(X_{2}^{+}, T\right)=Y_{2}^{\prime}\left(X_{2}^{-}, T\right) \\
& -E I Y_{1}^{\prime \prime \prime}\left(X_{1}^{-}, T\right)=-E\left(I_{t}+I_{b}\right) Y_{2}^{\prime \prime \prime}\left(X_{1}^{+}, T\right) \\
& -E I Y_{3}^{\prime \prime \prime}\left(X_{2}^{-}, T\right)=-E\left(I_{t}+I_{b}\right) Y_{2}^{\prime \prime \prime}\left(X_{2}^{+}, T\right)
\end{aligned}
$$

The compatibility conditions of bending moments at $X_{1}$ and $X_{2}$ are

$$
\begin{aligned}
-E I Y_{1}^{\prime \prime}\left(X_{1}^{-}, T\right)= & -\left(1+c_{1}^{*}\right)\left(E I_{t}+E I_{b}\right) Y_{2}^{\prime \prime}\left(X_{1}^{+}, T\right) \\
& +\left(\frac{E A_{t} A_{b} h^{2}}{4 L_{2}\left(A_{t}+A_{b}\right)}\right)\left(Y_{1}^{\prime}\left(X_{1}^{-}, T\right)-Y_{3}^{\prime}\left(X_{2}^{+}, T\right)\right) \\
-E I Y_{3}^{\prime \prime}\left(X_{2}^{-}, T\right)= & -\left(1+c_{2}^{*}\right)\left(E I_{t}+E I_{b}\right) Y_{2}^{\prime \prime}\left(X_{2}^{+}, T\right) \\
& +\left(\frac{E A_{t} A_{b} h^{2}}{4 L_{2}\left(A_{t}+A_{b}\right)}\right)\left(Y_{1}^{\prime}\left(X_{1}^{-}, T\right)-Y_{3}^{\prime}\left(X_{2}^{+}, T\right)\right)
\end{aligned}
$$

The following nondimensional quantities are introduced: 


$$
y=\frac{Y}{L}, x=\frac{X}{L}, x_{k}=\frac{X_{k}}{L}, l_{1}=\frac{L_{1}}{L}, l_{2}=\frac{L_{2}}{L}, l_{3}=\frac{L_{3}}{L}
$$

Let $t=\sqrt{\frac{\rho A L^{4}}{E I}}$; equations (35) and (36) become

$$
\begin{aligned}
& E I \frac{\partial^{4} y_{k}(x, t)}{\partial x^{4}}+\rho A \frac{\partial^{2} y_{k}(x, t)}{\partial t^{2}}=0, \quad x_{k-1}<x<x_{k}, \quad k=1,3 \\
& E\left(I_{t}+I_{b}\right) \frac{\partial^{4} y_{2}(x, t)}{\partial x^{4}}+\rho A \frac{\partial^{2} y_{2}(x, t)}{\partial t^{2}}=0, x_{1}<x<x_{2}
\end{aligned}
$$

Let $y_{k}(x, t)=w_{k}(x) e^{j \omega t}$, where $\omega$ is the natural frequency, and $w_{k}(x)$ is the mode shape of the $k$ th segment of the beam, equations (41) and (42) become

$$
w_{k}^{\prime \prime \prime \prime}(x)-\lambda_{k}^{4} w_{k}(x)=0, \quad x_{k-1}<x<x_{k}, \quad k=1,2,3
$$

where

$$
\lambda_{1}^{4}=\frac{\rho A \omega^{2} L^{4}}{E I}, \quad \lambda_{2}^{4}=\frac{\rho A \omega^{2} L^{4}}{E\left(I_{t}+I_{b}\right)}, \quad \lambda_{3}^{4}=\frac{\rho A \omega^{2} L^{4}}{E I}
$$

Let $\Lambda=\frac{E A_{t} A_{b} h^{2}}{4 L_{2}\left(A_{t}+A_{b}\right)}, \quad I_{l}=\left(1+c_{1}^{*}\right)\left(I_{t}+I_{b}\right), \quad$ and $\quad I_{r}=\left(1+c_{2}^{*}\right)\left(I_{t}+I_{b}\right) ; \quad$ the continuity and compatibility conditions in equations (38) and (39) become

$$
\begin{aligned}
& w_{1}\left(x_{1}^{-}\right)=w_{2}\left(x_{1}^{+}\right) \\
& w_{2}\left(x_{2}^{-}\right)=w_{3}\left(x_{2}^{+}\right) \\
& w_{1}^{\prime}\left(x_{1}^{-}\right)=w_{2}^{\prime}\left(x_{1}^{+}\right) \\
& w_{2}^{\prime}\left(x_{2}^{-}\right)=w_{3}^{\prime}\left(x_{2}^{+}\right) \\
& -E I w_{1}^{\prime \prime \prime}\left(x_{1}^{-}\right)=-E\left(I_{t}+I_{b}\right) w_{2}^{\prime \prime \prime}\left(x_{1}^{+}\right) \\
& -E I w_{3}^{\prime \prime \prime}\left(x_{2}^{-}\right)=-E\left(I_{t}+I_{b}\right) w_{2}^{\prime \prime \prime}\left(x_{2}^{+}\right) \\
& -E I w_{1}^{\prime \prime}\left(x_{1}^{-}\right)=-E I_{l} w_{2}^{\prime \prime}\left(x_{1}^{+}\right)+\Lambda\left(w_{1}^{\prime}\left(x_{1}^{-}\right)-w_{3}^{\prime}\left(x_{2}^{+}\right)\right) \\
& -E I w_{3}^{\prime \prime}\left(x_{2}^{+}\right)=-E I_{r} w_{2}^{\prime \prime}\left(x_{2}^{+}\right)+\Lambda\left(w_{1}^{\prime}\left(x_{1}^{-}\right)-w_{3}^{\prime}\left(x_{2}^{+}\right)\right)
\end{aligned}
$$

The general solution of equation (43) for each segment of the beam is 


$$
\begin{aligned}
w_{k}(x)= & A_{k}^{\prime} \sin \lambda_{k}\left(x-x_{k-1}\right)+B_{k}^{\prime} \cos \lambda_{k}\left(x-x_{k-1}\right) \\
& +C_{k}^{\prime} \sinh \lambda_{k}\left(x-x_{k-1}\right)+D_{k}^{\prime} \cosh \lambda_{k}\left(x-x_{k-1}\right), \quad x_{k-1}<x<x_{k}, k=1,2,3
\end{aligned}
$$

where $A_{k}^{\prime}, B_{k}^{\prime}, C_{k}^{\prime}$, and $D_{k}^{\prime}$ are unknown constants associated with the $k$ th segment of the beam for each natural frequency. By equation (45), one can relate the unknown constants associated with the $(k+1)$ th segment of the beam to those associated with the $k$ th segment:

$$
\left[\begin{array}{cccc}
t_{11}^{k} & t_{12}^{k} & t_{13}^{k} & t_{14}^{k} \\
t_{21}^{k} & t_{22}^{k} & t_{23}^{k} & t_{24}^{k} \\
t_{31}^{k} & t_{32}^{k} & t_{33}^{k} & t_{34}^{k} \\
t_{41}^{k} & t_{42}^{k} & t_{43}^{k} & t_{44}^{k}
\end{array}\right]\left\{\begin{array}{l}
A_{k}^{\prime} \\
B_{k}^{\prime} \\
C_{k}^{\prime} \\
D_{k}^{\prime}
\end{array}\right\}=\left[\begin{array}{llll}
t_{11}^{k+1} & t_{12}^{k+1} & t_{13}^{k+1} & t_{14}^{k+1} \\
t_{21}^{k+1} & t_{22}^{k+1} & t_{23}^{k+1} & t_{24}^{k+1} \\
t_{31}^{k+1} & t_{32}^{k+1} & t_{33}^{k+1} & t_{34}^{k+1} \\
t_{41}^{k+1} & t_{42}^{k+1} & t_{43}^{k+1} & t_{44}^{k+1}
\end{array}\right]\left\{\begin{array}{l}
A_{k+1}^{\prime} \\
B_{k+1}^{\prime} \\
C_{k+1}^{\prime} \\
D_{k+1}^{\prime}
\end{array}\right\}
$$

Let

$$
\begin{aligned}
& \mathbf{T}_{1}=\left[\begin{array}{cc}
\sin \lambda_{1} l_{1} & \cos \lambda_{1} l_{1} \\
\lambda_{1} \Lambda \cos \lambda_{1} l_{1}-E I \lambda_{1}^{2} \sin \lambda_{1} l_{1} & -\lambda_{1} \Lambda \sin \lambda_{1} l_{1}-E I \lambda_{1}^{2} \cos \lambda_{1} l_{1} \\
-E I \lambda_{1}^{3} \cos \lambda l_{1} & E I \lambda_{1}^{3} \sin \lambda l_{1} \\
\lambda_{1} \cos \lambda_{1} l_{1} & -\lambda_{1} \sin \lambda_{1} l_{1}
\end{array}\right. \\
& \sinh \lambda_{1} l_{1} \quad \cosh \lambda_{1} l_{1} \\
& \lambda_{1} \Lambda \cosh \lambda_{1} l_{1}+E I \lambda_{1}^{2} \sinh \lambda_{1} l_{1} \quad E I \lambda_{1}^{2} \cosh \lambda_{1} l_{1}+\lambda_{1} \Lambda \sinh \lambda_{1} l_{1} \\
& E I \lambda_{1}^{3} \cosh \lambda l_{1} \quad E I \lambda_{1}^{3} \sinh \lambda l_{1} \\
& \lambda_{1} \cosh \lambda_{1} l_{1} \quad \lambda_{1} \sinh \lambda_{1} l_{1} \\
& \mathbf{T}_{2}=\left[\begin{array}{cccc}
0 & 1 & 0 & 1 \\
\lambda_{2} \Lambda \cos \lambda_{2} l_{2} & -E I_{l} \lambda_{2}^{2}-\lambda_{2} \Lambda \sin \lambda_{2} l_{2} & \lambda_{2} \Lambda \cosh \lambda_{2} l_{2} & \lambda_{2} \Lambda \sinh \lambda_{2} l_{2}+E I_{l} \lambda_{2}^{2} \\
-\lambda_{2}{ }^{3} E\left(I_{t}+I_{b}\right) & 0 & \lambda_{2}^{3} E\left(I_{t}+I_{b}\right) & 0 \\
\lambda_{2} & 0 & \lambda_{2} & 0
\end{array}\right]
\end{aligned}
$$




$$
\mathbf{T}_{4}=\left[\begin{array}{cccc}
0 & 1 & 0 & 1 \\
-\lambda_{3} \Lambda & -E I \lambda_{3}^{2} & -\lambda_{3} \Lambda & E I \lambda_{3}^{2} \\
-E I \lambda_{3}^{3} & 0 & E I \lambda_{3}^{3} & 0 \\
\lambda_{3} & 0 & \lambda_{3} & 0
\end{array}\right]
$$

Then

$$
\begin{gathered}
\left\{\begin{array}{l}
A_{2}^{\prime} \\
B_{2}^{\prime} \\
C_{2}^{\prime} \\
D_{2}^{\prime}
\end{array}\right\}=\mathbf{T}_{2}^{-1} \mathbf{T}_{1}\left\{\begin{array}{l}
A_{1}^{\prime} \\
B_{1}^{\prime} \\
C_{1}^{\prime} \\
D_{1}^{\prime}
\end{array}\right\} \\
\left\{\begin{array}{l}
A_{3}^{\prime} \\
B_{3}^{\prime} \\
C_{3}^{\prime} \\
D_{3}^{\prime}
\end{array}\right\}=\mathbf{T}_{4}^{-1} \mathbf{T}_{3} \mathbf{T}_{2}^{-1} \mathbf{T}_{1}\left\{\begin{array}{l}
A_{1}^{\prime} \\
B_{1}^{\prime} \\
C_{1}^{\prime} \\
D_{1}^{\prime}
\end{array}\right\}
\end{gathered}
$$

Furthermore, the boundary conditions in equation (37) are reduced to

$$
\begin{aligned}
& w(0)=0, \quad w^{\prime}(0)=0 \\
& w^{\prime \prime}(1)=0, \quad w^{\prime \prime \prime}(1)=0
\end{aligned}
$$

Applying the boundary conditions in equation (54) to equation (46) yields

$$
A_{1}^{\prime}+C_{1}^{\prime}=0, \quad B_{1}^{\prime}+D_{1}^{\prime}=0
$$

Applying the boundary conditions in equation (55) to equation (46) yields

$$
\begin{aligned}
& -\lambda_{3}^{2} A_{3}^{\prime} \sin \lambda_{3}\left(l_{3}\right)-\lambda_{3}^{2} B_{3}^{\prime} \cos \lambda_{3}\left(l_{3}\right)+\lambda_{3}^{2} C_{3}^{\prime} \sinh \lambda_{3}\left(l_{3}\right)+\lambda_{3}^{2} D_{3}^{\prime} \cosh \lambda_{3}\left(l_{3}\right)=0 \\
& -\lambda_{3}^{3} A_{3}^{\prime} \cos \lambda_{3}\left(l_{3}\right)+\lambda_{3}^{3} B_{3}^{\prime} \sin \lambda_{3}\left(l_{3}\right)+\lambda_{3}^{3} C_{3}^{\prime} \cosh \lambda_{3}\left(l_{3}\right)+\lambda_{3}^{3} D_{3}^{\prime} \sinh \lambda_{3}\left(l_{3}\right)=0
\end{aligned}
$$

equations (57) and (58) can be written in the matrix form

$$
\mathbf{B}\left\{\begin{array}{l}
A_{3}^{\prime} \\
B_{3}^{\prime} \\
C_{3}^{\prime} \\
D_{3}^{\prime}
\end{array}\right\}=\left\{\begin{array}{l}
0 \\
0
\end{array}\right\}
$$

where 


$$
\mathbf{B}=\left[\begin{array}{cccc}
-\sin \lambda_{3}\left(l_{3}\right) & -\cos \lambda_{3}\left(l_{3}\right) & \sinh \lambda_{3}\left(l_{3}\right) & \cosh \lambda_{3}\left(l_{3}\right) \\
-\cos \lambda_{3}\left(l_{3}\right) & \sin \lambda_{3}\left(l_{3}\right) & \cosh \lambda_{3}\left(l_{3}\right) & \sinh \lambda_{3}\left(l_{3}\right)
\end{array}\right]
$$

Substituting equation (53) into (59) yields

$$
\left\{\begin{array}{l}
0 \\
0
\end{array}\right\}=\mathbf{B}\left\{\begin{array}{l}
A_{3}^{\prime} \\
B_{3}^{\prime} \\
C_{3}^{\prime} \\
D_{3}^{\prime}
\end{array}\right\}=\mathbf{B} \mathbf{T}_{4}^{-1} \mathbf{T}_{3} \mathbf{T}_{2}^{-1} \mathbf{T}_{1}\left\{\begin{array}{l}
A_{1}^{\prime} \\
B_{1}^{\prime} \\
C_{1}^{\prime} \\
D_{1}^{\prime}
\end{array}\right\}
$$

Let

$$
\mathbf{R}=\mathbf{B} \mathbf{T}_{4}^{-1} \mathbf{T}_{3} \mathbf{T}_{2}^{-1} \mathbf{T}_{1}=\left[\begin{array}{llll}
R_{11} & R_{12} & R_{13} & R_{14} \\
R_{21} & R_{22} & R_{23} & R_{24}
\end{array}\right]
$$

Use of equations (56), (61), and (62) yields

$$
\left[\begin{array}{ll}
R_{11}-R_{13} & R_{12}-R_{14} \\
R_{21}-R_{23} & R_{22}-R_{24}
\end{array}\right]\left\{\begin{array}{l}
A_{1}^{\prime} \\
B_{1}^{\prime}
\end{array}\right\}=\left\{\begin{array}{l}
0 \\
0
\end{array}\right\}
$$

Existence of a non-trivial solution of equation (63) requires

$$
\operatorname{det}\left[\begin{array}{ll}
R_{11}-R_{13} & R_{12}-R_{14} \\
R_{21}-R_{23} & R_{22}-R_{24}
\end{array}\right]=0
$$

which is the frequency equation of the three-segment beam model $f(\omega)=0$. The natural frequencies of the beam $\omega_{n}$, where $n$ is the mode number, can be obtained from the frequency equation using Newton-Raphson method. The initial guesses of $\omega_{n}$ are chosen to be close to the roots of the frequency equation, which can be obtained by plotting the function $f(\omega)$ and finding the approximate roots of $f(\omega)=0$. By equations (48)-(53), (61), and (64), and assuming $B_{1}^{\prime}$, has an arbitrary known value, one can obtain all the other constants of $A_{k}^{\prime}, B_{k}^{\prime}, C_{k}^{\prime}$, and $D_{k}^{\prime}(k=1,2,3)$ in $w_{k}(x)$ in equation (46). The normalized mode shapes of the beam are defined by

$$
\hat{w}_{n}(x)=\frac{w_{n}(x)}{\max \left(w_{n}(x)\right)}
$$




\section{Numerical results}

\subsection{Verification of the $J$-integrals}

To verify the analytical expressions of the $J$-integrals in equations (28) and (32), nondimensional numerical results of the $J$-integrals surrounding the two crack tips are calculated using commercial FE software [57]. Two-dimensional (2D) singular elements PLANE183, which are six-node shell elements with two degrees of freedom at each node, are used around the crack tips of the cantilever beam. The other parts of the beam are modeled using 2D plane strain solid elements PLANE42. The FE model of a cracked cantilever beam is shown in Figure 4. The parameters used are $P=1, E=1$, $h / L=0.05$, and $L_{2} / L=0.1$, with various ratios of $X_{c} / L$ and $h_{1} / h$; the Poisson's ratio of the beam is assumed to be $v=0.3$. Table 1 shows the comparison of the nondimensional plane stress results of the $J$-integrals corresponding to the right and left crack tips from the FE method, which are obtained by dividing the calculated plane strain ones by $1-v^{2}$, and the nondimensional results of the $J$-integrals corresponding to the right and left crack tips defined by $\widehat{J}=\left(E h^{3} /\left(P^{2} L^{2}\right)\right) J^{r}=\left(E h^{3} /\left(P^{2} L^{2}\right)\right) J^{l}$, where $J^{r}$ and $J^{l}$ are determined by equations (28) and (32), respectively. Since there are small differences between the FE results of the $J$-integrals corresponding to the right and left crack tips, their mean values are compared with the analytical results. The numerical results show that the differences between the two methods are less than $3.8 \%$, which demonstrates that equations (28) and (32) are valid for Euler-Bernoulli beam segments. Since the top and bottom segments are assumed to have the same transverse displacements and they can slide over each other in the axial direction 
except at their ends, the stress intensity factors $K_{\mathrm{II}}$ from the FE and proposed methods are compared to verify the proposed $J$-integral approach, as shown in Table 2 . The results show that the differences between the two methods are less than $2 \%$, which further demonstrates that the proposed $J$-integral approach is valid for Euler-Bernoulli beam segments.

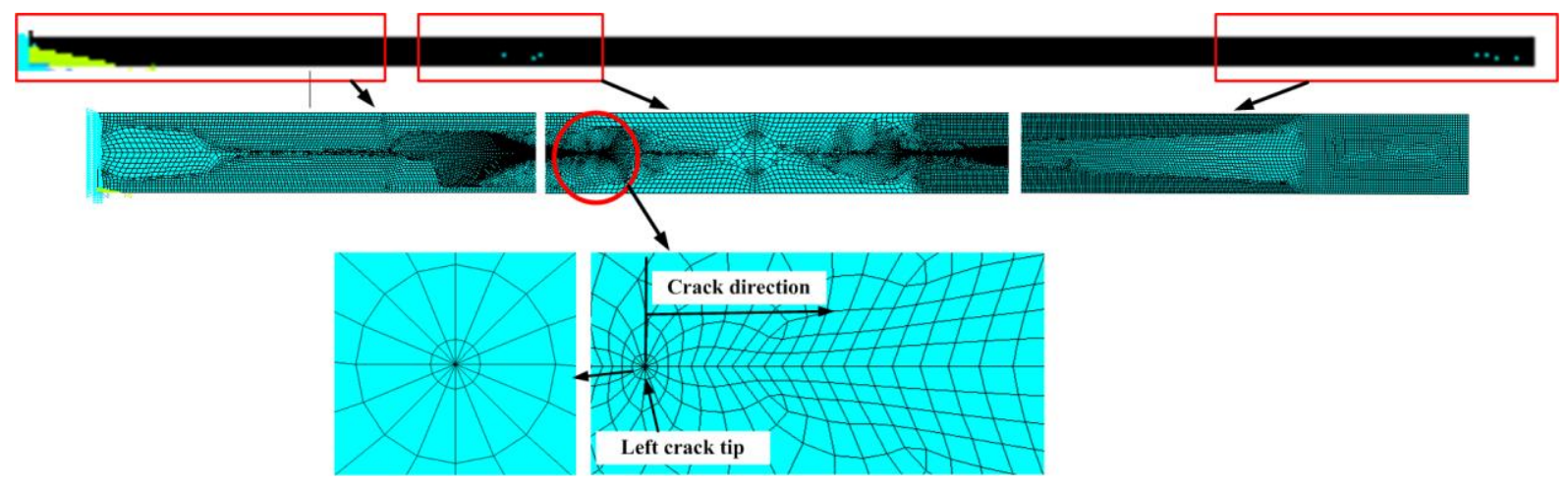

Figure 4. FE model of a cracked cantilever beam.

Table 1. Comparison of the nondimensional results of the $J$-integrals corresponding to the right and left crack tips from the FE method and equations (28) and (32), respectively.

\begin{tabular}{|c|c|c|c|c|c|c|}
\hline \multirow{2}{*}{$X_{C} / L$} & \multirow{2}{*}{$h_{1} / h$} & \multicolumn{3}{|c|}{ FE method } & \multirow{2}{*}{ Equations (28) and (32) } & \multirow{2}{*}{ Difference (\%) } \\
\cline { 3 - 5 } & & Right & Left & Mean value & & 3.77 \\
\hline 0.4 & 0.3 & 0.0257 & 0.0273 & 0.0265 & 0.0255 & 2.67 \\
\hline 0.5 & 0.3 & 0.0258 & 0.0266 & 0.0262 & 0.0255 & 3.41 \\
\hline 0.6 & 0.3 & 0.0265 & 0.0262 & 0.0264 & 0.0255 & 3.26 \\
\hline 0.4 & 0.4 & 0.0386 & 0.0412 & 0.0399 & 0.0386 & 0.77 \\
\hline 0.5 & 0.4 & 0.0390 & 0.0387 & 0.0389 & 0.0386 & 1.28 \\
\hline 0.6 & 0.4 & 0.0392 & 0.0390 & 0.0391 & 0.0386 & \\
\hline
\end{tabular}


Table 2. Comparison of the stress intensity factors $K_{\mathrm{II}}$ at the right and left crack tips from the FE and proposed methods.

\begin{tabular}{|c|c|c|c|c|c|c|}
\hline \multirow{2}{*}{$X_{C} / L$} & \multirow{2}{*}{$h_{1} / h$} & \multicolumn{3}{|c|}{ FE method } & \multirow{2}{*}{$\begin{array}{c}\text { Proposed } \\
\text { method }\end{array}$} & \multirow{2}{*}{ Difference (\%) } \\
\cline { 3 - 5 } & & Right & Left & Mean value & 14.973 & 1.90 \\
\hline 0.4 & 0.3 & 15.031 & 15.492 & 15.263 & 143 & 1.34 \\
\hline 0.5 & 0.3 & 15.060 & 15.292 & 15.177 & 14.973 & 1.63 \\
\hline 0.6 & 0.3 & 15.263 & 15.177 & 15.220 & 14.973 & 1.64 \\
\hline 0.4 & 0.4 & 18.421 & 19.032 & 18.729 & 18.421 & 0.32 \\
\hline 0.5 & 0.4 & 18.516 & 18.445 & 18.481 & 18.421 & 0.64 \\
\hline 0.6 & 0.4 & 18.563 & 18.516 & 18.540 & 18.421 & \\
\hline
\end{tabular}

\subsection{Effect of local flexibilities at crack tips on natural frequencies and mode shapes of a cracked cantilever beam}

Consider a cracked cantilever beam with $L=600 \mathrm{~mm}, h=b=10 \mathrm{~mm}, E=2.06 \times 10^{11}$ $\mathrm{N} / \mathrm{m}^{2}$, and $\rho=7800 \mathrm{~kg} / \mathrm{m}^{3}$. The first three natural frequencies of the beam without the crack are $\omega_{01}=23.060 \mathrm{~Hz}, \omega_{02}=144.516 \mathrm{~Hz}$, and $\omega_{03}=404.649 \mathrm{~Hz}$. The ratios of the first three natural frequencies of the three-segment beam and the corresponding one without local flexibilities at the crack tips to those of the beam without the crack are compared, as shown in Table 3. While the natural frequencies from the three-segment beam models with and without local flexibilities at the crack tips decrease with the length and depth of the crack, the differences between the natural frequencies from the three-segment beam models with and without local flexibilities at the crack tips range from $0.048 \%$ to $2.949 \%$. 
Table 3. Comparison between the first three natural frequencies from the three-segment beam models with and without local flexibilities at the crack tips.

\begin{tabular}{|c|c|c|c|c|c|}
\hline \multirow{2}{*}{$X_{1} / L \times h_{1} / h$} & \multirow{2}{*}{$L_{2} / L$} & \multirow{2}{*}{ Three-segment beam models } & \multicolumn{3}{|c|}{ Natural frequency ratios } \\
\hline & & & $\omega_{1} / \omega_{01}$ & $\omega_{2} / \omega_{02}$ & $\omega_{3} / \omega_{03}$ \\
\hline \multirow{3}{*}{$0.25 \times 0.4$} & \multirow{3}{*}{0.1} & With local flexibilities & 0.861 & 0.958 & 0.881 \\
\hline & & Without local flexibilities & 0.857 & 0.959 & 0.879 \\
\hline & & Difference $(\%)$ & 0.431 & 0.068 & 0.224 \\
\hline \multirow{3}{*}{$0.25 \times 0.4$} & \multirow{3}{*}{0.15} & With local flexibilities & 0.835 & 0.914 & 0.868 \\
\hline & & Without local flexibilities & 0.820 & 0.915 & 0.863 \\
\hline & & Difference $(\%)$ & 1.837 & 0.048 & 0.551 \\
\hline \multirow{3}{*}{$0.2 \times 0.2$} & \multirow{3}{*}{0.2} & With local flexibilities & 0.902 & 0.957 & 0.920 \\
\hline & & Without local flexibilities & 0.890 & 0.961 & 0.915 \\
\hline & & Difference $(\%)$ & 1.435 & 0.385 & 0.577 \\
\hline \multirow{3}{*}{$0.2 \times 0.3$} & \multirow{3}{*}{0.2} & With local flexibilities & 0.844 & 0.930 & 0.885 \\
\hline & & Without local flexibilities & 0.820 & 0.936 & 0.878 \\
\hline & & Difference $(\%)$ & 2.949 & 0.582 & 0.814 \\
\hline
\end{tabular}

Figure 5 shows the comparison of the first three normalized mode shapes of the cracked cantilever beam with $X_{1} / L=0.25, h_{1} / h=0.4$, and $L_{2} / L=0.1$ from the three-segment beam models with and without local flexibilities at the crack tips (Figures 5(a)-(c)) and their single-level SWT decomposition detail coefficients [58,59] calculated using the MATLAB program SWT (Figures 5(d)-(f)); the crack is located between points $\mathrm{A}$ and $\mathrm{B}$, and the normalized mode shapes from the three-segment beam model without local flexibilities at the crack tips can be obtained from the results in Section 3 with $c_{1}=c_{2}=0$. The differences between the first three normalized mode shapes from the two models in Figures 5(a)-(c) increase with the crack length, as well as those between the corresponding SWT decomposition detail coefficients of the three mode shapes in Figures 5(d)-(f). The effects of the local flexibilities at the crack tips on the first three mode shapes and their SWT decomposition detail coefficients increase with the crack length. Hence, the local flexibilities at the crack 
tips should be considered in the cantilever beam model.

(a)

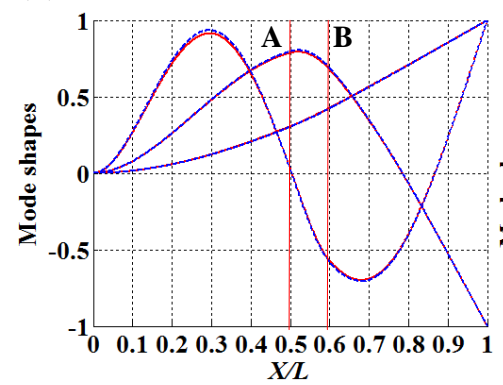

(d)

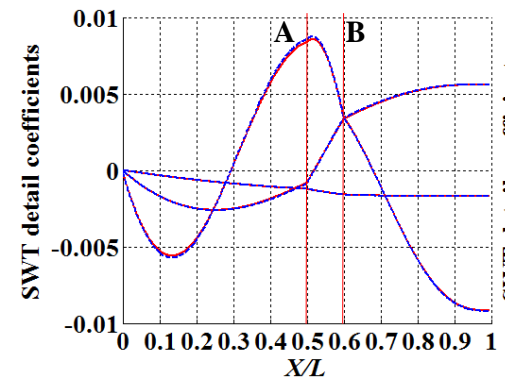

(b)

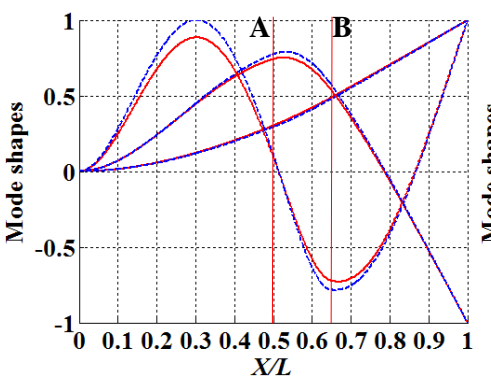

(e)

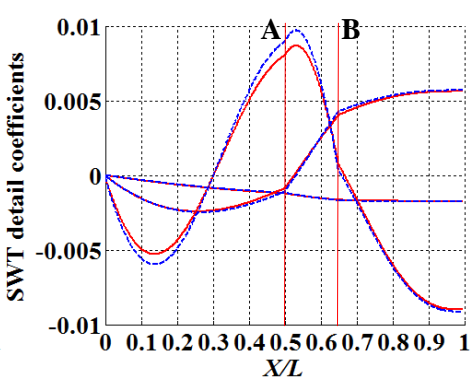

(c)

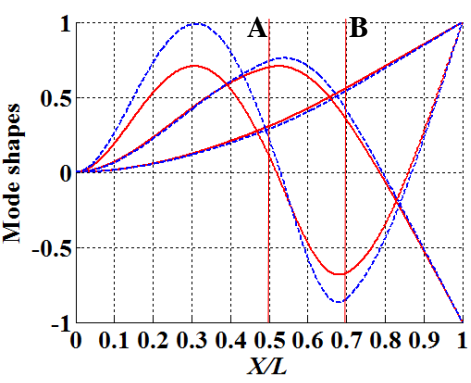

(f)

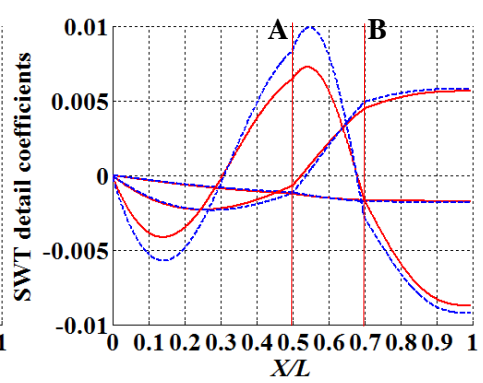

Figure 5. Comparison of the first three normalized mode shapes from the three-segment beam models with (-) and without (---) local flexibilities at the crack tips and their SWT decomposition detail coefficients: (a) $X_{1} / L=0.5, L_{2} / L=0.1$, and $h_{1} / h=0.4$; (b) $X_{1} / L=0.5, L_{2} / L=0.15$, and $h_{1} / h=0.4$; (c) $X_{1} / L=0.5, L_{2} / L=0.2$, and $h_{1} / h=0.4$; (d) $X_{1} / L=0.5, L_{2} / L=0.1$, and $h_{1} / h=0.4$; (e) $X_{1} / L=0.5, L_{2} / L=0.15$, and $h_{1} / h=0.4$; and (f) $X_{1} / L=0.5, L_{2} / L=0.2$, and $h_{1} / h=0.4$.

\subsection{Effects of the crack length, location, and depth on the mode shapes of the cracked cantilever beam}

The effects of the crack length, location, and depth on single-level SWT decomposition detail coefficients of the first three normalized mode shapes of the cracked cantilever beam are shown in Figures 6-8, respectively. The crack lengths and locations can be clearly and directly identified from kinks in the single-level SWT 
decomposition detail coefficients of the first three normalized mode shapes of the beam, where the cracks are located between points a and b, points a and c, and points $\mathrm{a}$ and $\mathrm{d}$ in Figure 6; between points a and d, points $\mathrm{b}$ and $\mathrm{e}$, and $\mathrm{c}$ and $\mathrm{f}$ in Figure 7; and between points $\mathrm{a}$ and $\mathrm{b}$ in Figure 8 . It can be seen that the amplitudes of the kinks slightly increase with the crack depth in Figure 8.

(a)

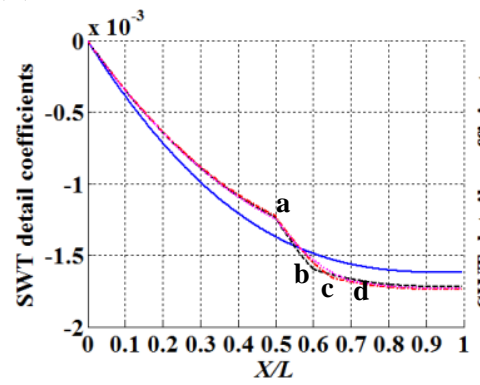

(b)

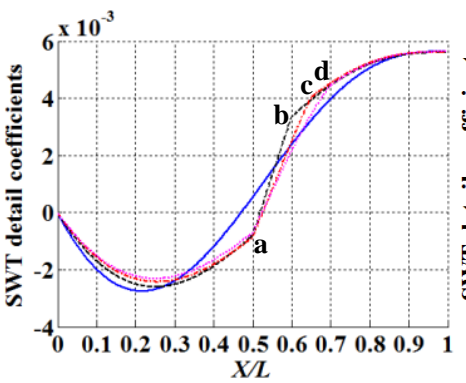

(c)

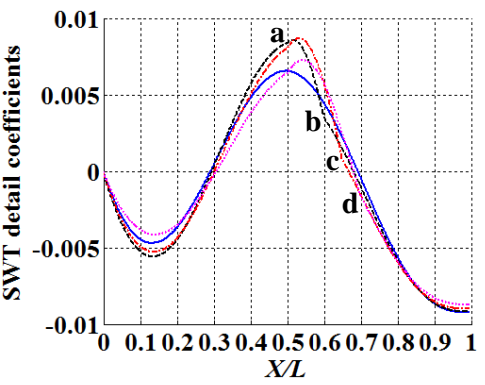

Figure 6. Effect of the crack length on SWT decomposition detail coefficients of the first three normalized mode shapes of the beam with and without the crack: - , the beam without the crack; ---, $X_{1} / L=0.5, L_{2} / L=0.1$, and $h_{1} / h=0.4 ;-.-, X_{1} / L=0.5$, $L_{2} / L=0.15$, and $h_{1} / h=0.4 ; \ldots, X_{1} / L=0.5, L_{2} / L=0.2$, and $h_{1} / h=0.4$. (a) The first mode, (b) the second mode, and (c) the third mode.

(a)

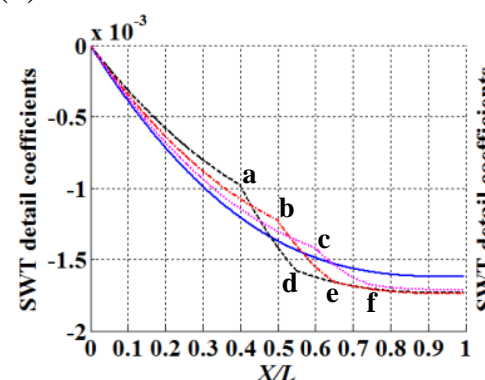

(b)

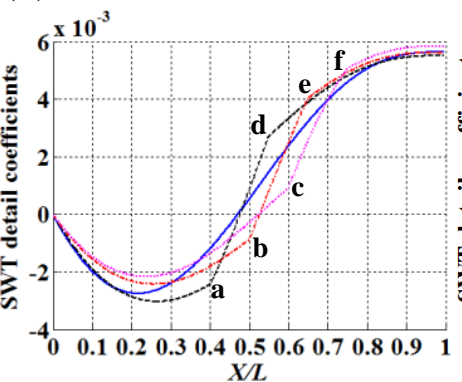

(c)

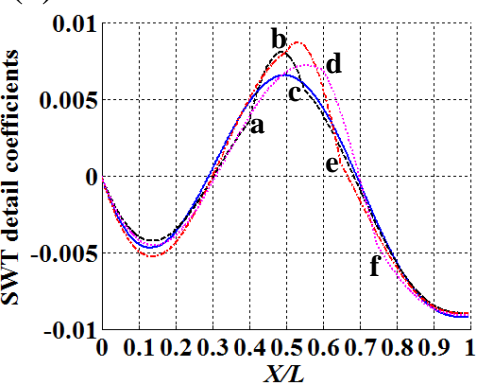

Figure 7. Effect of the crack location on SWT decomposition detail coefficients of the first three normalized mode shapes of the beam with and without the crack: - , the beam without the crack; ---, $X_{1} / L=0.4, L_{2} / L=0.15$, and $h_{1} / h=0.4 ;-.-, X_{1} / L=0.5$, 
$L_{2} / L=0.15$, and $h_{1} / h=0.4 ; \ldots, X_{1} / L=0.6, L_{2} / L=0.15$, and $h_{1} / h=0.4$. (a) The first mode,

(b) the second mode, and (c) the third mode.

(a)

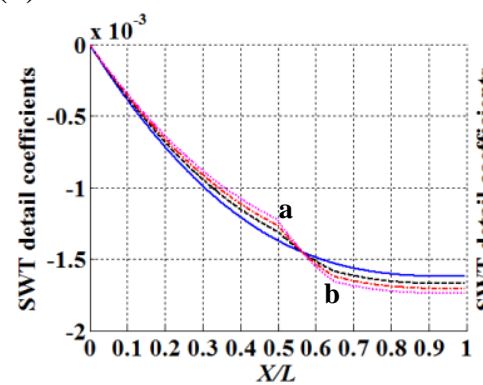

(b)

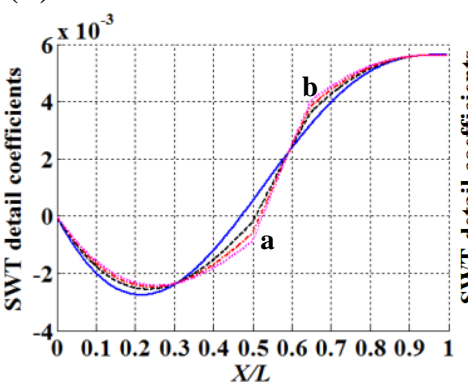

(c)

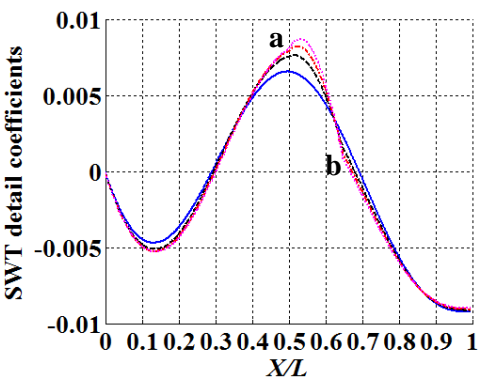

Figure 8. Effect of the crack depth on SWT decomposition detail coefficients of the first three normalized mode shapes of the beam with and without the crack: - , the beam without the crack; ---, $X_{1} / L=0.5, L_{2} / L=0.15$, and $h_{1} / h=0.2 ;-$ -,$X_{1} / L=0.5$, $L_{2} / L=0.15$, and $h_{1} / h=0.3 ; \ldots, X_{l} / L=0.5, L_{2} / L=0.15$, and $h_{1} / h=0.4$. (a) The first mode, (b) the second mode, and (c) the third mode.

\section{Experimental validation and numerical verification}

To validate the three-segment beam model, a cracked acrylonitrile butadiene styrene beam of length $111.4 \mathrm{~mm}$, height $5.2 \mathrm{~mm}$, and width $10.5 \mathrm{~mm}$ is made by a 3D printer, as shown in Figure 9(a). The length, height, and width of a horizontal, rectangular crack are $16.6 \mathrm{~mm}, 0.3 \mathrm{~mm}$, and $10.5 \mathrm{~mm}$, respectively, as shown in Figure 9(b). The distance between the left end of the crack and the fixed end of the beam is $53.1 \mathrm{~mm}$, and that between the top surface of the crack and the top surface of beam is $2.6 \mathrm{~mm}$.

(a)
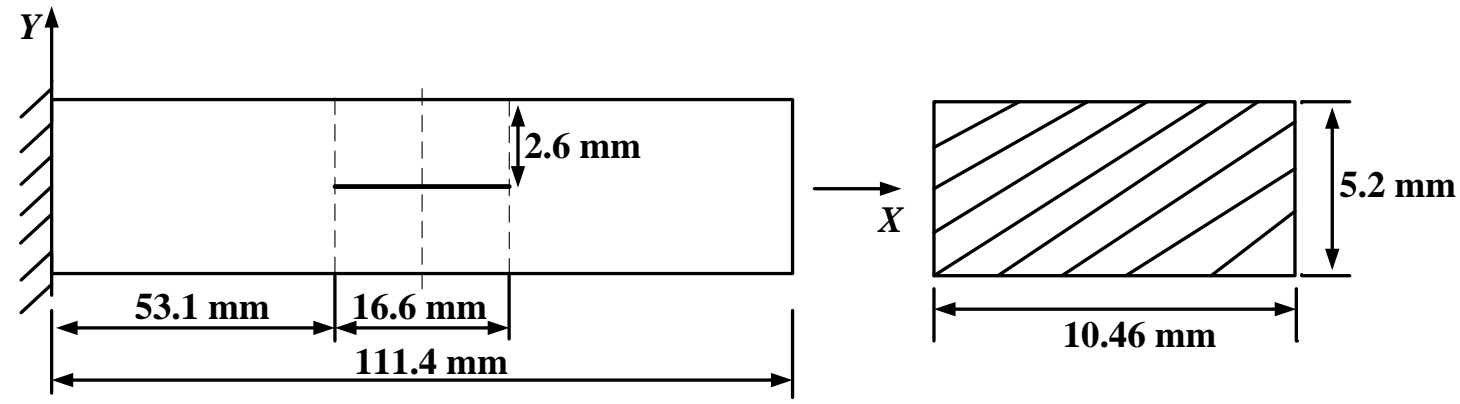
(b)

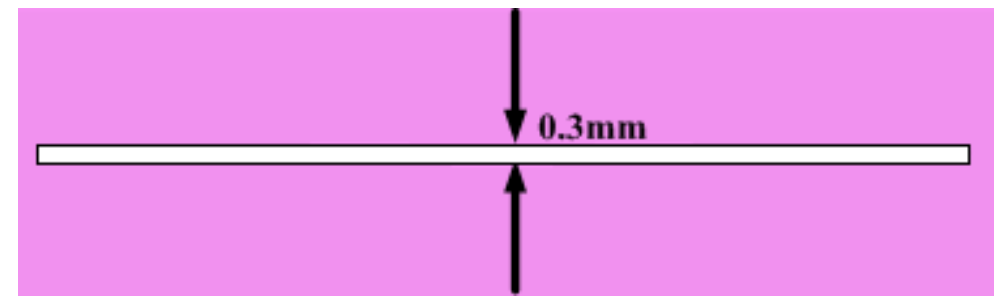

Figure 9. (a) Dimensions of a cantilever beam with an embedded crack, (b) an enlarged view of the crack region.

An experimental setup is shown in Figure 10. An operational modal analysis with non-contact excitation and measurement is performed on the cracked beam. An electric speaker is used to generate acoustic excitation to the beam. Two Doppler laser vibrometers are used to measure the response of the beam: Laser 1 and Laser 2 measure velocities of measurement points and the velocity of a reference point on the beam, respectively. There are totally 129 measurement points on the beam, which are evenly distributed along the length of beam. The third natural frequency and mode shape of the cracked beam are measured using Operational PloyMax of LMS Test. Lab Rev. 9b.

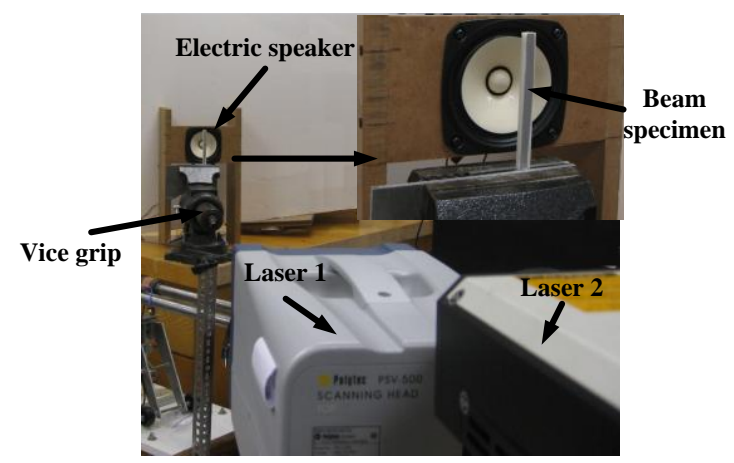

Figure 10. An experimental setup for the cracked beam using operational modal analysis.

The third natural frequencies form the experiment, the FE model, and the three-segment beam model are $1608 \mathrm{~Hz}, 1604.3 \mathrm{~Hz}$, and $1600.7 \mathrm{~Hz}$, respectively. The 
difference between the third natural frequency from the FE model and the experiment is $-0.25 \%$, and that between the three-segment beam and the experiment is $-0.45 \%$. The fourth natural frequency form the experiment, the FE model, and the three-segment beam model are $3124.2 \mathrm{~Hz}, 3179 \mathrm{~Hz}$, and $2966.8 \mathrm{~Hz}$, respectively. The difference between the natural frequency from the FE model and the experiment is $1.72 \%$, and that between the three-segment beam and the experiment is $-5.03 \%$. Figure 11 shows the third and fourth normalized mode shapes from the experiment, the FE model, and the three-segment beam model and their SWT decomposition detail coefficients. The third and fourth normalized mode shapes and their SWT decomposition detail coefficients from the experiment agree very well with those from the FE model, and they are similar in shape to those from the three-segment beam model. The spatial regions with abrupt changes of the SWT decomposition detail coefficients of the third and fourth normalized mode shape from the experiment, the FE model, and the three-segment beam model correspond to the crack region, as shown in Figures 11(c) and (d), which can be used to identify the crack length and location. The results from the experiment and the FE model validate and verify to some extent the three-segment beam model, respectively. Note that the results from the FE model are overall closer to those from the experiment than the three-segment beam model. One reason is that there is a non-zero height of the crack in the experiment and the FE model, which is not considered in the three-segment beam model. Note also that the proposed analytical method is useful even though the FE method is available. For example, such an analytical model can be easily implemented 
and run on a broad range of computational platforms, requiring minimal computational resources such as dedicated software, while also executed at minimal computational costs compared with the computational resources and costs required to run a fully tested FE model that can yield reliable convergent solutions.

(a)

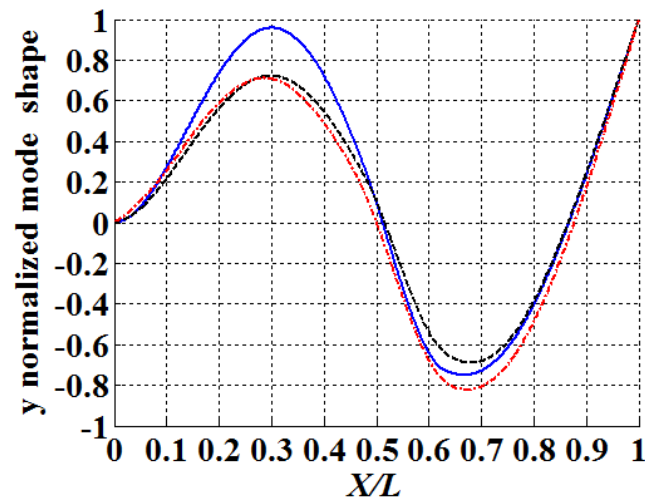

(c)

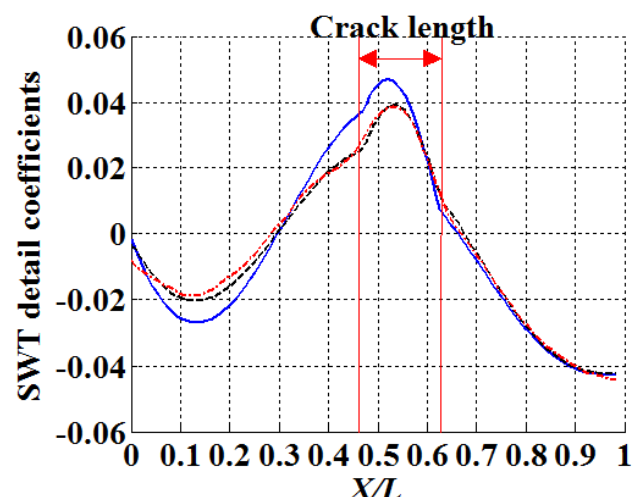

(b)

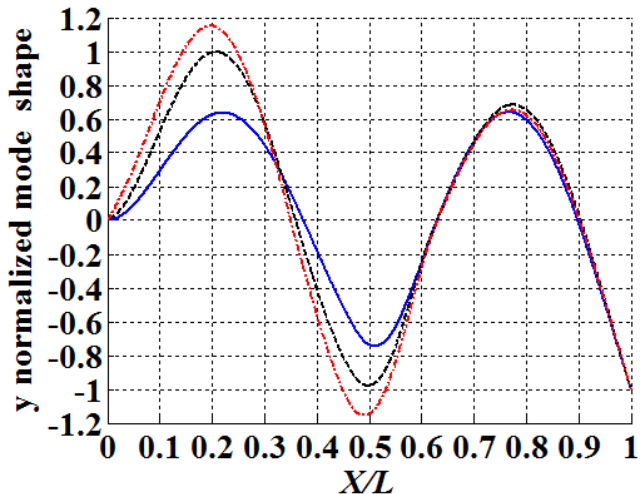

(d)

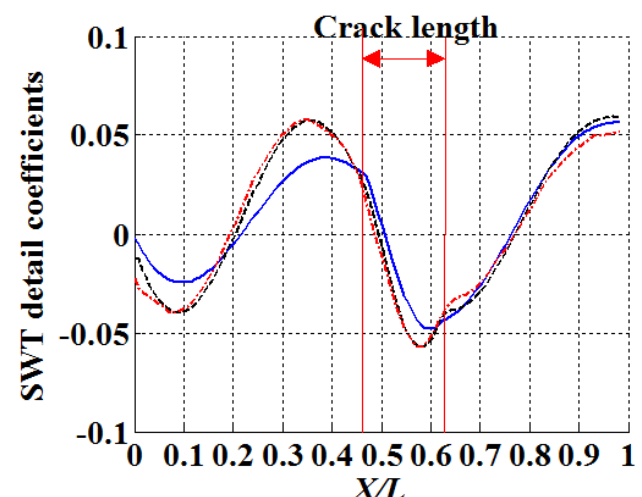

Figure 11. The third normalized mode shapes from the experiment $(-.-)$, the FE model (- - ), and the three-segment beam model (-) and their SWT decomposition detail coefficients: (a) the third mode shape; (b) the fourth mode shape; (c) SWT decomposition detail coefficients of the third mode shape; and (d) SWT decomposition detail coefficients of the fourth mode shape.

\section{Conclusion}

A new three-segment beam model with local flexibilities at crack tips is 
developed to investigate the vibration of a cantilever beam with a closed, fully embedded horizontal crack. The effect of the crack is modeled by local flexibilities at the crack tips and a reduced bending moment of the middle segment of the beam containing the crack. The compliances at the crack tips are analytically determined using a $J$-integral approach. The natural frequencies and mode shapes of the three-segment beam with local flexibilities at the crack tips are derived using compatibility conditions at the crack tips and the transfer matrix method. The following conclusions can be obtained from this study:

1) The values of the $J$-integrals along the contours of the left and right parts of the beam are the same for Euler-Bernoulli beam segments. The equivalent rotational flexibility of the cross-section of the beam at the left crack tip is larger than that at the right tip. The differences between the $J$-integral results from the analytical and FE methods are less than $3.8 \%$. The differences between the stress intensity factors $K_{\text {II }}$ from the analytical and FE methods are less than $2 \%$.

2) The differences between the natural frequencies from the three-segment beam models with and without local flexibilities at the crack tips range from $0.048 \%$ to $2.949 \%$.

3) The differences between the first three normalized mode shapes from the three-segment beam models with and without local flexibilities at the crack tips increase with the crack length, as well as those between the corresponding SWT decomposition detail coefficients of the three mode shapes. The local flexibilities at the crack tips should be considered in the cantilever beam model. 
4) The regions of abrupt changes of single-level SWT decomposition detail coefficients of normalized mode shapes of the cracked cantilever beam correspond to the crack region, which can be used to identify the length and location of a closed, fully embedded horizontal crack.

\section{Acknowledgement}

The authors are grateful for the financial support provided by the National Natural Science Foundation of China under Grant Numbers 51475053 and 11442006, the National Science Foundation under Grant Numbers CMMI-1000830, CMMI-1229532, and CMMI-1335024, the University of Maryland Baltimore County Directed Research Initiative Fund Program, and the Fundamental Research Funds for the Central Universities.

\section{Appendix: Determination of the $J$-integral along the contour FEDCBA of the left part of the beam in Figure 2(a)}

The $J$-integral along the contour FEDCBA of the left part of the beam in Figure 2(a) is the sum of line integrals along segments FE, ED, DC, CB, and BA of the contour:

$$
J^{l}=J_{B A}^{l}+J_{C B}^{l}+J_{D C}^{l}+J_{E D}^{l}+J_{F E}^{l}
$$

where the superscript $l$ denotes the left part. For segments $\mathrm{CB}$ and $\mathrm{ED}, d Y=0$ and $T_{i}=0$; hence

$$
J_{C B}^{l}=0, \quad J_{E D}^{l}=0
$$


For segment BA, one has

$$
J_{B A}^{l}=\int_{B A}\left(W_{A B}^{l} d y-T_{i} \frac{\partial u_{i}}{\partial X} d s\right)=\int_{B A}\left(-W_{A B}^{l} d s-\left(T_{1} \frac{\partial u_{1}^{l}}{\partial X}+T_{2} \frac{\partial u_{2}^{l}}{\partial X}\right) d s\right)
$$

where

$$
W_{B A}^{l}=\frac{1}{2} \sigma_{x x 2}^{l} \varepsilon_{x x 2}^{l}+\frac{1}{2} \tau_{x y 2}^{l} \gamma_{x y 2}^{l}
$$

in which $\sigma_{x x 2}^{l}$ and $\tau_{x y 2}^{l}$ are the normal and shear stresses on segment BA, respectively, $\varepsilon_{x x 2}^{l}$ and $\gamma_{x y 2}^{l}$ are the normal and shear strains of segment BA, respectively, and the subscript 2 denotes the stress and strain components associated with segment BA. They are given by

$$
\begin{aligned}
& \sigma_{x x 2}^{l}=\frac{N_{2}}{A_{b}}-\frac{M_{2}}{I_{b}} Y, \varepsilon_{x x 2}^{l}=\frac{\partial u_{1}}{\partial X}=\frac{N_{2}}{E A_{b}}-\frac{M_{2}}{E I_{b}} Y \\
& \tau_{x y 2}^{l}=\frac{P_{2} S(Y)}{I_{b} t}, \gamma_{x y 2}^{l}=\frac{\tau_{x y 2}^{l}}{G}=\frac{\partial u_{1}^{l}}{\partial Y}+\frac{\partial u_{2}^{l}}{\partial X}=\frac{P_{2} S(Y)}{G I_{b} t}
\end{aligned}
$$

where $u_{1}^{l}$ and $u_{2}^{l}$ are the displacements of the beam segment of $X_{1} X_{2}$ in the $X$ and $Y$ directions, respectively. The relationship between the displacements in the $X$ and $Y$ directions is

$$
u_{1}^{l}=u_{1}^{0 l}-\frac{\partial u_{2}^{0 l}}{\partial X} Y
$$

where the superscript 0 denotes deflections along the centroidal axis of the bottom segment of $X_{1} X_{2}$. Differentiating equation (A6) with respect to $Y$ yields

$$
\frac{\partial u_{1}^{l}}{\partial Y}=-\frac{\partial^{2} u_{2}^{0 l}}{\partial X \partial Y} Y-\frac{\partial u_{2}^{0 l}}{\partial X}=-\frac{\partial u_{2}^{0 l}}{\partial X}=-\frac{\partial u_{2}^{l}}{\partial X}
$$

where $\frac{\partial^{2} u_{2}^{0 l}}{\partial X \partial Y}=0$. For the cracked cantilever beam, the rotational angle of the cross-section BA is assumed to be the sum of the rotational angle of the cross-section at $X_{1}$ relative to that at $X_{0}$ and the rotational angle of the cross-section at $X_{\mathrm{c}}$ relative to 
that at $X_{1}$ :

$$
\frac{\partial u_{2}^{l}}{\partial X}=-\frac{P L_{1}^{2}}{2 E I}-\frac{P\left(L-L_{1}\right) L_{1}}{E I}-\frac{P_{2} a^{2}}{2 E I_{b}}+\frac{M_{2} a}{E I_{b}}
$$

Then

$$
\gamma_{x y 2}^{l}=\frac{\partial u_{2}^{l}}{\partial X}=-\frac{P L_{1}^{2}}{2 E I}-\frac{P\left(L-L_{1}\right) L_{1}}{E I}-\frac{P_{2} a^{2}}{2 E I_{b}}+\frac{M_{2} a}{E I_{b}}
$$

The traction vector components for segment BA are given by

$$
T_{1}=\sigma_{11} n_{1}+\sigma_{12} n_{2}=-\sigma_{11}, T_{2}=\sigma_{21} n_{1}+\sigma_{22} n_{2}=-\sigma_{21}
$$

Use of equations (A4)-(A9) in equation (A3) yields

$$
\begin{aligned}
J_{B A}^{l} & =\int_{A B} \frac{1}{2} \sigma_{x x 1}^{l} \varepsilon_{x x 1}^{l} d Y+\int_{A B} \tau_{x y 1}^{l} \frac{\partial u_{1}^{l}}{\partial X} d Y \\
& =\frac{N_{2}{ }^{2}}{2 E A_{b}}+\frac{M_{2}{ }^{2}}{2 E I_{b}}+\frac{P P_{2} L_{1}^{2}}{2 E I}+\frac{P P_{2}\left(L-L_{1}\right) L_{1}}{E I}+\frac{P_{2}^{2} a^{2}}{2 E I_{b}}+\frac{P_{2} M_{2} a}{E I_{b}}
\end{aligned}
$$

Similarly, the line integral along segment FE is

$$
\begin{aligned}
J_{F E}^{l} & =\int_{F E} \frac{1}{2} \sigma_{x x 2}^{l} \varepsilon_{x x 2}^{l} d y+\int_{F E} \tau_{x y 2}^{l} \frac{\partial u_{2}^{l}}{\partial X} d Y \\
& =\frac{N_{1}^{2}}{2 E A_{t}}+\frac{M_{1}^{2}}{2 E I_{t}}+\frac{P P_{1} L_{1}^{2}}{2 E I}+\frac{P P_{1}\left(L-L_{1}\right) L_{1}}{E I}+\frac{P_{1}^{2} a^{2}}{2 E I_{t}}+\frac{P_{1} M_{1} a}{E I_{t}}
\end{aligned}
$$

For segment DC,

$$
J_{D C}^{l}=\int_{D C} W_{D C}^{l} d Y-T_{i} \frac{\partial u_{i}}{\partial X} d s=\int_{D C}\left(W_{D C}^{l}-\left(T_{1} \frac{\partial u_{1}^{l}}{\partial X}+T_{2} \frac{\partial u_{2}^{l}}{\partial X}\right)\right) d s
$$

where

$$
W_{D C}^{l}=\frac{1}{2} \sigma_{x x 4}^{l} \varepsilon_{x x 4}^{l}+\frac{1}{2} \tau_{x y 4}^{l} \gamma_{x y 4}^{l}
$$

in which the subscript 4 denotes the stress and strain components associated with segment DC. The normal stress, normal strain, shear stress, and shear strain for segment DC are 


$$
\begin{aligned}
& \sigma_{x x 4}^{l}(Y)=\frac{M Y}{I}, \varepsilon_{x x 4}^{l}(Y)=\frac{\sigma_{x x 4}^{l}}{E}=\frac{\partial u_{1}^{l}}{\partial X}=\frac{M Y}{E I} \\
& \tau_{x x 4}^{l}=\frac{P S(Y)}{I t_{1}}, \gamma_{x x 4}^{l}=\frac{\tau_{x x 4}^{l}}{G}=\frac{\partial u_{1}^{l}}{\partial Y}+\frac{\partial u_{2}^{l}}{\partial X}=\frac{P S(Y)}{G I t_{1}}=0
\end{aligned}
$$

where $M=-P L$ is the bending moment at the cross-section at $X_{0}$. The traction vector components $T_{i}$ for segment DC are

$$
T_{1}=\sigma_{11} n_{1}+\sigma_{12} n_{2}=\sigma_{11}, T_{2}=\sigma_{21} n_{1}+\sigma_{22} n_{2}=\sigma_{21}
$$

Use of equations (A15) and (A16) in equation (13) yields

$$
\begin{aligned}
J_{D C}^{l} & =\int_{D C}\left(W_{D C}^{l}-\left(T_{1} \frac{\partial u_{1}^{l}}{\partial X}+T_{2} \frac{\partial u_{2}^{l}}{\partial X}\right)\right) d s \\
& =\int_{D C}\left(\frac{1}{2} \sigma_{x x 4}^{l} \varepsilon_{x x 4}^{l}+\frac{1}{2} \tau_{x x 4}^{l} \gamma_{x x 4}^{l}-\left(\sigma_{11} \frac{\partial u_{1}^{l}}{\partial X}+\sigma_{21} \frac{\partial u_{2}^{l}}{\partial X}\right)\right) d s \\
& =-\int_{D C} \frac{1}{2} \sigma_{x x 4}^{l} \varepsilon_{x x 4}^{l} d s=-\frac{6 L^{2}}{E t_{1} h^{3}} P^{2}
\end{aligned}
$$

Substituting equations (A2), (A3), (A11), (A12), and (A17) into equation (A1) yields

$$
J^{l}=J_{B A}^{l}+J_{C B}^{l}+J_{D C}^{l}+J_{E D}^{l}+J_{F E}^{l}=\left(\frac{h^{3}}{h_{1}^{3}+\left(h-h_{1}\right)^{3}}-1\right) \frac{6 a^{2} P^{2}}{E h^{3}}
$$

\section{References}

[1] Fan W and Qiao PZ (2011) Vibration-based damage identification methods: a review and comparative study. Structural Health Monitoring 10(1): 83-111.

[2] Jassim ZA, Ali NN, Mustapha F and Jalil NAA (2013) A review on the vibration analysis for a damage occurrence of a cantilever beam. Engineering Failure Analysis 31: 442-461.

[3] Ostachowicz WM and Krawczuk M (1991) Analysis of the effect of cracks on the 
natural frequencies of a cantilever beam. Journal of Sound and Vibration 150(2): 191-201.

[4] Wang KH, Inman DJ and Farrar CR (2005) Modeling and analysis of a cracked composite cantilever beam vibrating in coupled bending and torsion. Journal of Sound and Vibration 284: 23-49.

[5] Hearn G (1991) Modal analysis for damage detection in structures. Journal of Structural Engineering 117(10): 3042-3063.

[6] Ren WX and Roeck GD (2002) Structural damage identification using modal data I: simulation verification. Journal of Structural Engineering 128(1): 87-95.

[7] Ren WX and Roeck GD (2002) Structural damage identification using modal data II: test verification. Journal of Structural Engineering 128(1): 96-104.

[8] Owolabi GM, Swamidas ASJ and Seshadri R (2003) Crack detection in beams using changes in frequencies and amplitudes of frequency response functions. Journal of Sound and Vibration 265: 1-22.

[9] Nahvi $\mathrm{H}$ and Jabbari M (2005) Crack detection in beams using experimental modal data and finite element model. International Journal of Mechanical Sciences 47: 1477-1497.

[10] Khiem NT and Lien TV (2004) Multi-crack detection for beam by the natural frequencies. Journal of Sound and Vibration 273(1): 175-184.

[11] Ouahabi A, Thomas M and Lakis AA (2006) Detection of damages in beams and composite plates by harmonic excitation and time-frequency analysis. 3rd 
European Workshop on Structural Health Monitoring, Granada, Spain.

[12] Yuen MF (1985) A numerical study of the eigenparameters of a damaged cantilever. Journal of Sound and Vibration 103: 301-310.

[13] Rizos PF and Aspragathos N (1990) Identification of crack location and magnitude in a cantilever beam from the vibrating mode. Journal of Sound and Vibration 138(3): 381-388.

[14] Narkis Y (1994) Identification of crack location in vibrating simply supported beams. Journal of Sound and Vibration 172(4): 549-558.

[15] Nandwana BP and Maiti SK (1997) Detection of the location and size of a crack in stepped cantilever beams based on measurements of natural frequencies. Journal of Sound and Vibration 203(3): 435-446.

[16] Ishak SI, Liu GR, Shang HM and Lim SP (2002) Non-destructive evaluation of forizontal crack detection in beams using transverse impact. Journal of Sound and Vibration 252(2): 343-360.

[17] Li B, Chen XF, Ma JX and He ZJ (2005) Detection of crack location and size in structures using wavelet finite element methods. Journal of Sound and Vibration 285(4): 767-782.

[18] Lin HP and Chang SC (2006) Forced response of cracked cantilever beams subjected to a concentrated moving load. International Journal of Mechanical Sciences 48: 1456-1463.

[19] Alsabbagh ASY, Abuzeid OM and Dado MH (2009) Simplified stress correction 
factor to study the dynamic behavior of a cracked beam. Applied Mathematical Modelling 33(1): 127-139.

[20] Rubio L (2009) An efficient method for crack identification in simply supported Euler-Bernoulli beams. ASME Journal of Vibration and Acoustics 131: 051001.

[21] Behzad M, Meghdari A and Ebrahimi A (2005) A new approach for vibration analysis of cracked beam. International Journal of Engineering 18(4): 319-330.

[22] Behzad M, Ebrahimi A and Meghdari A (2010) A continuous vibration theory for beams with a vertical edge crack. Transaction B Mechanical Engineering 17(3): 194-204.

[23] Wang CS and Lee LT (2012) Modified and simplified sectional flexibility of a cracked beam. Journal of Applied Mathematics 2012: 1-16.

[24] Lu XB, Liu JK and Lu ZR (2013) A two-step approach for crack identification in beam. Journal of Sound and Vibration 332: 282-293.

[25] Chondros TG, Dimarogonas AD and Yao J (1998) A continuous cracked beam vibration theory. Journal of Sound and Vibration 215: 17-34.

[26] Shifrin EI and Ruotolo R (1999) Natural frequencies of a beam with an arbitrary number of cracks. Journal of Sound and Vibration 222(3): 409-423.

[27] Lin HP, Chang SC and Wu JD (2002) Beam vibrations with arbitrary number of cracks. Journal of Sound and Vibration 258(5): 987-999.

[28] Kim JT and Stubbs N (2003) Crack detection in beam-type structures using frequency data. Journal of Sound and Vibration 259(1): 145-160. 
[29] Chang CC and Chen LW (2005) Detection of the location and size of cracks in the multiple cracked beam by spatial wavelet based approach. Mechanical Systems and Signal Processing 19(1): 139-155.

[30] Patil DP and Maiti SK (2005) Experimental verification of a method of detection of multiple cracks in beams based on frequency measurements. Journal of Sound and Vibration 281(1): 439-451.

[31] Mujumdar PM and Suryanaryan (1988) Flexural vibrations of beams with delaminations. Journal of Sound and Vibration 125(3): 441-461.

[32] Shen MHH and Grady JE (1992) Free vibration of delaminated beams. AIAA Journal 30(5): 1361-1370.

[33] Lee J, Haftka RT and Griffin OH (1994) Detecting delaminations in a composite beam using anti-optimization. Structural Optimization 8(2-3): 93-100.

[34] Wang Q and Deng XM (1999) Damage detection with spatial wavelets. International Journal of Solids and Structures 36: 3443-3468.

[35] Luo H and Hanagud S (2000) Dynamics of delaminated beams. International Journal of Solids and Structures 37: 1501-1519.

[36] Zou Y, Tong L and Steven GP (2000) Vibration-based model-dependent damage (delamination) identification and health monitoring for composite structures-a review. Journal of Sound and Vibration 230(2): 357-378.

[37] Lee JH (2000) Free vibration analysis of delaminated composite beams. Computers and Structures 74: 121-129. 
[38] Wang J and Qiao P (2004) Novel beam analysis of end notched flexure specimen for mode- II fracture. Engineering Fracture Mechanics 71: 219-231.

[39] Wang Q, Moslehy F and Nicholson DW (2005) Stability analysis of a delaminated beam subjected to follower compression. AIAA Journal 43(9): 2052-2059.

[40] Della CN and Shu DW (2007) Free vibration analysis of delaminated biomaterial beams. Composite Structures 80: 212-220.

[41] Wildy SJ, Kotousov AG, Cazzolato BS and Codrington JD (2010) New damage detection technique based on governing differential equations of continuum mechanics, Part I: out-of-plane loading. Proceedings of the 6th Australasian Congress on Applied Mechanics (ACAM 6), Perth, Australia.

[42] Wu N and Wang Q (2010) Repair of vibrating delaminated beam structures using piezoelectric patches. Smart Materials and Structures 19: 035027.

[43] Wang Q and Wu N (2011) Detecting the delaminations location of a beam with a wavelet transform: an experimental study. Smart Materials and Structures 20: 012002.

[44] Erdelyi NH and Hashemi SM (2012) A dynamic stiffness element for free vibration analysis of delaminated layered beams. Modelling and Simulation in Engineering 2012: 492415.

[45] Qiao PZ and Chen FL (2012) On the improved dynamic analysis of delaminated beams. Journal of Sound and Vibration 331: 1143-1163. 
[46] Qian XD, Cao MS and Su ZQ (2012) A hybrid particle swarm optimization (PSO)-simplex algorithm for damage identification of delaminated beams. Mathematical Problems in Engineering 2012: 1-11.

[47] Fang X (2013) The mechanics of an elastically deforming cantilever beam with an embedded sharp crack and subjected to an end transverse loading. PhD Thesis, University of Maryland, Baltimore County, USA.

[48] Rice JR (1968) A path independent integral and the approximate analysis of strain concentration by notches and cracks. Journal of Applied Mechanics 35: 379-386.

[49] Meirovitch L. Principles and techniques of vibrations. New Jersey: Prentice Hall; 1997.

[50] Orhan S (2007) Analysis of free and forced vibration of a cracked cantilever beam. NDT\&E International 40: 443-450.

[51] Zhong SC, Yadiji SO and Ding K (2008) Response-only method for damage detection of beam-like structures using high accuracy frequencies with auxiliary mass spatial probing. Journal of Sound and Vibration 311: 1075-1099.

[52] Lee J (2009) Identification of multiple cracks in a beam using vibration amplitude. Journal Sound and Vibration 326(1-2): 205-212.

[53] Masoud AA and Said SA (2009) A new algorithm for crack location in a rotating Timoshenko beam. Journal of Vibration and control 15(10): 1541-1561.

[54] Kim DG and Lee SB (2010) Structural damage identification of a cantilever 
beam using excitation force level control. Mechanical Systems and Signal Processing 24(6): 1814-1830.

[55] Cao M, Ye L and Zhou L (2011) Sensitivity of fundamental mode shape and static deflection for damage identification in cantilever beams. Mechanical Systems and Signal Processing 25(2): 630-643.

[56] Wu N and Wang Q (2011) Experimental studies on damage detection of beam structures with wavelet transform. International Journal of Engineering Science 49(3): 253-261.

[57] ANSYS Theory Reference (2007) ANSYS Release 11.0: ANSYS, Inc..

[58] Zhong S and Oyadiji SO (2007) Crack detection in simply-supported beams without baseline model parameter by stationary wavelet transform. Mechanical Systems and Signal Processing 21(4): 1853-1884.

[59] Zhong SC, Guo JQ, Yao LG, Zhuang YZ and Lu HD (2012) Wavelet-based damage localization in plate-like structures. Proceedings of the ASME 2012 International Mechanical Engineering Congress \& Exposition, Texas, USA.

[60] Sharf I (1999) Nonlinear strain measures, shape functions and beam elements for dynamics of flexible beams. Multibody System Dynamics 3: 189-205.

[61] Hellan K (1985) Introduction to fracture mechanics. US: McGraw-Hill Inc..

[62] Dimarogonas AD (1996) Vibration of cracked structures: a state of the art review. Engineering Fracture Mechanics 55(5): 831-857. 